\title{
HARD LEFSCHETZ THEOREM AND HODGE-RIEMANN RELATIONS FOR INTERSECTION COHOMOLOGY OF NONRATIONAL POLYTOPES
}

\author{
PAUL BRESSLER AND VALERY A. LUNTS
}

\begin{abstract}
The Hard Lefschetz theorem for intersection cohomology of nonrational polytopes was recently proved by K. Karu [Ka]. This theorem implies the conjecture of R. Stanley on the unimodularity of the generalized $h$-vector. In this paper we strengthen Karu's theorem by introducing a canonical bilinear form $(\cdot, \cdot)_{\Phi}$ on the intersection cohomology $I H(\Phi)$ of a complete fan $\Phi$ and proving the Hodge-Riemann bilinear relations for $(\cdot, \cdot)_{\Phi}$.
\end{abstract}

\section{INTRODUCTION}

For an $n$-dimensional convex polytope $Q \mathrm{R}$. Stanley ([S]) defined a set of integers $h(Q)=\left(h_{0}(Q), h_{1}(Q), \ldots, h_{n}(Q)\right)$ - the "generalized $h$ vector" - which are supposed to be the intersection cohomology Betti numbers of the toric variety $X_{Q}$ corresponding to $Q$. In case $Q \subset \mathbb{R}^{n}$ is a rational polytope the variety $X_{Q}$ indeed exists, and it is known $([\mathrm{S}])$ that $h_{i}(Q)=\operatorname{dim} I H^{2 i}\left(X_{Q}\right)$. Thus, for a rational polytope $Q$, the integers $h_{i}(Q)$ satisfy

(1) $h_{i}(Q) \geq 0$

(2) $h_{i}(Q)=h_{n-i}(Q)$ (Poincaré duality),

(3) $h_{0}(Q) \leq h_{1}(Q) \leq \ldots \leq h_{[n / 2]}(Q)$ (follows from the Hard Lefschetz theorem for projective algebraic varieties).

For an arbitrary convex polytope (more generally for an Eulerian poset) Stanley proved ([S], Theorem 2.4) the property 2 above. He conjectured that 1 and 3 also hold without the rationality hypothesis. This conjecture drew attention for the last fifteen years and many attempts had been made to prove it. A correct proof was recently given by K. Karu in [Ka]. The proof is based on the theory developed in $[\mathrm{BrLu}]$ and $[\mathrm{BBFK}]$, which, in particular, associates to an arbitrary convex polytope $Q$ its intersection cohomology space $I H(Q)$ with a Lefschetz operator $l$. In case $Q$ is rational, $I H(Q)=I H\left(X_{Q}\right)$ and $l$ is the

The authors were supported in part by the CRDF grant RM1-2405-MO-02. The second author was partially supported by the NSA grant MDA904-01-1-0020. 
multiplication by the Chern class of an ample line bundle on $X$; so $l$ satisfies the Hard Lefschetz theorem on $I H(Q)$. The similar Hard Lefschetz (HL) property of $l$ was conjectured for an arbitrary $Q$ in [BrLu], $[\mathrm{BBFK}]$. It was shown in $[\mathrm{BrLu}]$ the $(\mathrm{HL})$ property implies in particular the equality $\operatorname{dim} I H^{2 k}(Q)=h_{k}(Q)$, hence implies the Stanley's conjecture. The (HL) theorem was proved in [Ka].

The construction of the intersection cohomology $I H(Q)$ is based on the study of the equivariant geometry of the (nonexistent in general) toric variety $X_{Q}$ and was essentially given in [BeLu]. Namely, one first defines the "equivariant" intersection cohomology of $Q$ and then the ordinary $I H(Q)$. This equivariant part of the theory is indispensable.

More precisely, we work with the complete fan $\Phi$ which is dual to the polytope $Q$ (and denote $I H(\Phi)=I H(Q)$, etc.). We consider $\Phi$ as a (finite) topological space with subfans being the open subsets. There are two natural sheaves of rings on $\Phi$ : the constant sheaf $A_{\Phi}$ ( $A$ is the ring of global polynomial functions on the space of the fan) and the "structure sheaf" $\mathcal{A}_{\Phi}$, such that the stalk $\mathcal{A}_{\Phi, \sigma}$ at the cone $\sigma \in \Phi$ consists of polynomial functions on $\sigma$. (In case the space of the fan is the Lie algebra of a torus, the (evenly graded) algebra $A$ is naturally isomorphic to the cohomology ring of the classifying space of the torus and there is a close connection between $A_{\Phi^{-}}$-modules and equivariant sheaves on the corresponding toric variety $X$.)

The theory of $A_{\Phi}$-modules and $\mathcal{A}_{\Phi}$-modules was developed in [BrLu] and partially in [BBFK]. This includes Verdier duality, "equivariant perverse sheaves", decomposition theorem for subdivisions, etc. In particular there exists a minimal sheaf $\mathcal{L}_{\Phi}$ which plays the role of the equivariant intersection cohomology complex. The minimal sheaf is characterized by the property that it is an indecomposable (locally free) $\mathcal{A}_{\Phi}$-module with the stalk at the origin $\mathcal{L}_{\Phi, \underline{o}}=\mathbb{R}$. Then the "equivariant" intersection cohomology of $\Phi$ is the graded $A$-module $\Gamma\left(\Phi, \mathcal{L}_{\Phi}\right)$ and

$$
I H(\Phi):=\Gamma\left(\mathcal{L}_{\Phi}\right) / A^{+} \Gamma\left(\mathcal{L}_{\Phi}\right),
$$

where $A^{+} \subset A$ is the maximal ideal. It was proved in [BrLu], [BBFK] that $I H(\Phi)$ satisfies the Poincaré duality: $\operatorname{dim} I H^{n-k}(\Phi)=\operatorname{dim} I H^{n+k}(\Phi)$.

Note that $\Gamma\left(\mathcal{L}_{\Phi}\right)$ (and hence $I H(\Phi)$ ) is a module over $\mathcal{A}(\Phi)$ - the algebra of piecewise polynomial functions on $\Phi$. The following theorem was formulated as the main conjecture in $[\mathrm{BrLu}]$ and proved in $[\mathrm{Ka}]$.

Theorem 1.1. (HL) Assume that lis a strictly convex piecewise linear function on $\Phi$. Then the map

$$
l^{k}: I H^{n-k}(\Phi) \rightarrow I H^{n+k}(\Phi)
$$


is an isomorphism for all $k \geq 1$.

We show in this paper that by assuming the (HL) theorem for fans of dimension $\leq n-1$ we obtain a canonical pairing

$$
(\cdot, \cdot)_{\Phi}: I H(\Phi) \times I H(\Phi) \rightarrow \mathbb{R}(2 n)
$$

for fans $\Phi$ of dimension $n$. Then we prove the following theorem which is the analogue of the Hodge-Riemann bilinear relations in the (intersection) cohomology of algebraic varieties.

Theorem 1.2. (HR) Let $\Phi$ and $l$ be as in the (HL) theorem. Consider the primitive subspace

$$
\operatorname{Prim}_{l} I H^{n-k}(\Phi)=\operatorname{Ker}\left\{l^{k+1}: I H^{n-k}(\Phi) \rightarrow I H^{n+k+2}\right\} .
$$

Then for $0 \neq a \in \operatorname{Prim}_{l} I H^{n-k}(\Phi)$ we have

$$
(-1)^{\frac{n-k}{2}}\left(a, l^{k} a\right)_{\Phi}>0 .
$$

This theorem was also essentially proved by Karu, except he did not have a canonical pairing $(\cdot, \cdot)_{\Phi}$ and had to make choices at each step. This ambiguity makes the proof unnecessarily heavy and hard to follow. Actually the results of this paper imply that the pairing in $[\mathrm{Ka}]$ is independent of the choices made and coincides with the canonical one. For completeness we present the proofs of theorems (HL) and (HR), following the main ideas of $[\mathrm{Ka}]$. The proof proceeds by induction on the dimension and a reduction to the simplicial case which is known by McMullen [McM] (see also [Ti]).

We follow the idea in $[\mathrm{BBFK}]$ and try to develop all the notions and results not only for complete fans, but also for quasi-convex ones. (A fan $\Delta$ is quasi-convex if $\Gamma\left(\Delta, \mathcal{L}_{\Delta}\right)$ is a free $A$-module and hence it makes sense to define the intersection cohomology $I H(\Delta)$ the same way as for complete fans.) In particular, we define the canonical pairing $(\cdot, \cdot)$ for quasi-convex fans and then show that this pairing is compatible with various natural operations on fans such as subdivisions, embeddings of fans, etc.

Let us briefly describe the contents of the paper. In the second section we recall and collect some elementary facts about fans and polytopes which are used later. In the third section we recall the theory of sheaves on a fan according to $[\mathrm{BrLu}]$ and $[\mathrm{BBFK}]$, and formulate the (HL) and (HR) theorems. Section 4 is a review the "smooth" case corresponding to a simple polytope or a simplicial fan. Here we review Timorin's work in case of polytopes and Brion's work in case of fans. We then relate the two pictures in a natural way so that Timorin's Poincaré duality is identified with Brion's. In section 5 we 
define the canonical pairing on the intersection cohomology of quasiconvex fans and then in section 6 we show that for simplicial fans our pairing equals $n$ ! times Brion's (or Timorin's). This implies the (HL) and (HR) theorems in the simplicial case. Section 7 discusses compatibility of the canonical pairing with natural operations of fans. This makes the theory flexible and easy to use. In section 8 we obtain some immediate applications. Section 9 contains the proof of theorems $(\mathrm{HL})$ and $(\mathrm{HR})$. In section 10 we prove the Künneth formula for the intersection cohomology and derive the (HL) and (HR) theorems for the product of fans (these results are used in section 9).

We thank Kalle Karu for his useful remarks on the first version of this paper. Vladlen Timorin informed us that the definition of the polytope algebra $A(P)$ in section 4 and Proposition 4.3 are due to A.V. Pukhlikov and A.G. Khovanskii. He also claims that Proposition 4.2 was known to Minkowski.

\section{PRELIMINARIES ON FANS AND POLYTOPES}

Let us fix some terminology. Consider a linear space $V \simeq \mathbb{R}^{n}$. A (convex) cone in $V$ is the intersection of a finite number of closed halfspaces $\left\{L_{i} \geq 0\right\}$ where $L_{i} \in W:=V^{*}$. A face of a cone $\sigma$ is the intersection $\{L=0\} \cap \sigma$ where $L$ is a linear function on $V$ which is nonnegative on $\sigma$. A fan $\Phi$ in $V$ is a finite collection of cones with the following properties.

1. If $\sigma \in \Phi$ and $\tau \subset \sigma$ is a face, then $\tau \in \Phi$.

2. The intersection of two cones in $\Phi$ is a face of each.

3. The origin $\underline{o} \in \Phi$.

We denote by $\Phi^{\leq k} \subset \Phi$ the subfan consisting of all cones of dimension $\leq k$.

Given a fan $\Phi$ denote by $|\Phi| \subset V$ its support, i.e. the union of all cones in $\Phi$. We call $\Phi$ complete if $|\Phi|=V$.

Example 2.1. A fan $\Phi$ is simplicial if every cone $\sigma \in \Phi$ of dimension $k$ is a convex hull of $k$ rays.

For a subset $S \subset \Phi$ denote by $[S]$ the minimal subfan of $\Phi$ which contains $S$. Let $\sigma \in \Phi$. We denote

$$
\begin{aligned}
& \partial \sigma=[\sigma]-\sigma, \\
& \sigma^{0}=\sigma-|\partial \sigma|, \\
& \operatorname{St}(\sigma)=\{\tau \in \Phi \mid \sigma \subset \tau\}, \\
& \partial \operatorname{St}(\sigma)=[\operatorname{St}(\sigma)]-\operatorname{St}(\sigma), \\
& \operatorname{Link}(\sigma)=\{\tau \in \partial \operatorname{St}(\sigma) \mid \tau \cap \sigma=\underline{o}\}
\end{aligned}
$$

Sometimes we will be more specific and write $\operatorname{St}_{\Phi}(\sigma)$ instead of $\operatorname{St}(\sigma)$. 
We denote by $\langle\sigma\rangle$ the linear subspace of $V$ spanned by a cone $\sigma$.

Let $\Phi$ and $\Phi^{\prime}$ be fans in spaces $V$ and $V^{\prime}$ respectively. Let $\gamma:|\Phi| \rightarrow$ $\left|\Phi^{\prime}\right|$ be a homeomorphism which is linear on each cone in $\Phi$ and induces a bijection between $\Phi$ and $\Phi^{\prime}$. We call $\gamma$ a pl-isomorphism between $\Phi$ and $\Phi^{\prime}$ and say that these fans are pl-isomorphic.

Let $V_{1}, V_{2}$ be vector spaces with fans $\Delta$ and $\Sigma$ respectively. Consider the product $V=V_{1} \times V_{2}$ with the fan

$$
\Phi=\{\sigma+\tau \mid \sigma \in \Delta, \tau \in \Sigma\} .
$$

We call $\Phi=\Delta \times \Sigma$ the product fan.

Consider $A=\operatorname{Sym} V^{*}$ as a graded algebra, where linear functions have degree 2 . We denote by $\mathcal{A}(\Phi)$ the (also evenly graded) algebra of piecewise polynomial functions on $|\Phi|$, i.e. $f \in \mathcal{A}(\Phi)$ if $f_{\sigma}=\left.f\right|_{\sigma}$ is a polynomial for each $\sigma \in \Phi$.

A piecewise linear function $l \in \mathcal{A}^{2}(\Phi)$ is called strictly convex if for any two cones $\sigma$ and $\tau$ of dimension $n$ we have $l_{\sigma}(v)<l_{\tau}(v)$ for any $v \in \tau^{0}$. A complete fan $\Phi$ is called projective if it possesses a strictly convex piecewise linear function. Note that if $l$ is strictly convex, then so is $l+l^{\prime}$ for any $l^{\prime} \in A^{2}$.

Example 2.2. Let $P \subset W$ be a convex polytope of dimension $n$. For each face $F \subset P$ consider the cone $\sigma_{F} \subset V$ which is the dual to the cone in $W$ generated by vectors $f-p$ with $f \in F$ and $p \in P$. The collection of cones $\sigma_{F}$ is a complete fan in $V$, which is called the outer normal fan of $P$. We denote this fan $\Phi_{P}$. The assignment $F \mapsto \sigma_{F}$ is a bijective, order-reversing correspondence between faces of $P$ and cones of $\Phi_{P}$. Denote by $H_{P}$ the following function on $V$

$$
H_{P}(v)=\max _{y \in P}\langle y, v\rangle \text {. }
$$

Then $H_{P}$ is a piecewise linear function on $\Phi_{P}$, called the support function of $P$. It is strictly convex. Thus $\Phi_{P}$ is projective. Vice versa, given a projective fan $\Phi$ with a strictly convex piecewise linear function $l$ there exists a convex polytope $P \subset W$ such that $(\Phi, l)=\left(\Phi_{P}, H_{P}\right)$.

Example 2.3. Consider the product fan $\Phi=\Delta \times \Sigma$ as above. If $l_{1}$ and $l_{2}$ are piecewise linear strictly convex functions on $\Delta$ and $\Sigma$ respectively, then $l=l_{1}+l_{2}$ is strictly convex on $\Phi$.

Remark 2.4. Our main results imply that a strictly convex piecewise linear function $l$ on a complete fan behaves like the first Chern class of an ample line bundle on a projective variety. This may cause some confusion, because of the two opposite notions of strict convexity. Namely, if $l=H_{P}$ is the support function of a convex polytope on $\Phi=\Phi_{P}$, 
then $l$ is strictly lower convex: given two adjacent $d$-dimensional cones $\sigma, \tau \in \Phi$, we have $\left.l_{\sigma}\right|_{\tau}<l_{\tau}$. In [Da] however, a piecewise linear function which is the first Chern class of an ample line bundle, is strictly upper convex. The reason for this discrepancy is the fact that $\Phi_{P}$ is the outer normal fan of $P$, whereas Danilov uses the inner normal fan.

Let $\Phi$ and $\Psi$ be fans. We call $\Psi$ a subdivision of $\Phi$ if $|\Psi|=|\Phi|$ and every cone $\Psi$ is contained in a cone in $\Phi$. In this case we define a map $\pi: \Psi \rightarrow \Phi$ so that $\pi(\sigma)$ is the smallest cone in $\Phi$ which contains $\sigma$. Often we will refer to the map $\pi$ as a subdivision.

Example 2.5. Given a fan $\Phi$ and a cone $\sigma \in \Phi$ of dimension $>1$ choose a ray $\rho \subset \sigma^{0}$. Define a new fan

$$
\Psi:=\{\Phi-\operatorname{St}(\sigma)\} \cup\{\tau+\rho \mid \tau \in \partial \operatorname{St}(\sigma)\} .
$$

Then $\Psi$ is a subdivision of $\Phi$, called the star subdivision (defined by $\rho)$. Thus to obtain $\Psi$ from $\Phi$ one replaces $\operatorname{St}_{\Phi}(\sigma)$ by $\operatorname{St}_{\Psi}(\rho)$.

Remark 2.6. Given a star subdivision as above there is a one dimensional vector space of piecewise linear functions on $\Psi$ supported on $\operatorname{St}(\rho)$. Namely, such a function is determined by its restriction to $\rho$.

Definition 2.7. An edge (i.e. a 1-dimensional face) $\rho$ of a cone $\sigma$ is called free in $\sigma$ if all other edges of $\sigma$ are contained in one facet $\tau$, i.e.

$$
\sigma=\tau+\rho .
$$

A cone $\sigma$ is called deficient if it has no free edges.

Note that a fan is simplicial if and only if it contains no deficient cones.

Remark 2.8. In case of a star subdivision as in the previous example the 1-dimensional cone $\rho$ is free in every cone containing it.

Definition 2.9. For a fan $\Phi$ we define its "singular" subfan $\Phi^{s}$ to be the minimal subfan which contains all deficient cones in $\Phi$. So $\Phi^{s}=\emptyset$ if and only if $\Phi$ is simplicial.

In the notation of Example 2.5 let $\sigma \in \Phi^{s}$ be a cone of maximal dimension. By Remark 2.8 the resulting fan $\Psi$ will contain a smaller number of deficient cones than $\Phi$. This suggests a "desingularization" process of a fan, which ends when one obtains a simplicial fan. This strategy is used in $[\mathrm{Ka}]$ and we also follow it.

Definition 2.10. Let $\Phi$ be a fan and $\sigma \in \Phi$. We say that $\Phi$ has a local product structure at $\sigma$ if

$$
[\operatorname{St}(\sigma)]=\left\{\xi=\tau_{1}+\tau_{2} \mid \tau_{1} \in[\sigma], \tau_{2} \in \operatorname{Link}(\sigma)\right\}
$$


Example 2.11. Assume that a complete fan $\Phi$ has a local product structure at $\sigma \in \Phi$. Consider the projection $p: V \rightarrow \bar{V}=V /\langle\sigma\rangle$. Then $\Psi=p(\operatorname{Link}(\sigma))$ is a complete fan in $\bar{V}$ and $[\operatorname{St}(\sigma)]$ is pl-isomorphic to the product fan $\Psi \times[\sigma]$.

Lemma 2.12. Let $\Phi$ be a complete fan and $\sigma \in \Phi^{s}$ - a maximal cone in $\Phi^{s}$. Then $\operatorname{Link}(\sigma)$ is a simplicial fan and $\Phi$ has a local product structure at $\sigma$.

Proof. Claim: Every cone $\tau \in \operatorname{St}(\sigma)$ is of the form

$$
\tau=\sigma+\rho_{1}+\ldots+\rho_{\operatorname{dim}(\tau)-\operatorname{dim}(\sigma)},
$$

where the edges $\rho_{i}$ are free in $\tau$.

Indeed, let $\tau \in \operatorname{St}(\sigma)$ be a cone of the minimal dimension such that $\tau=\sigma+\rho_{1}+\ldots+\rho_{s}$ and $\operatorname{dim}(\tau)<\operatorname{dim}(\sigma)+s$. By definition of $\sigma$ the cone $\tau$ contains a free edge $\rho$, so that $\tau=\rho+\xi$ for a cone $\xi$. If $\rho \subset \sigma$, then $\sigma=\rho+\sigma \cap \xi$ and $\rho$ is free in $\sigma-$ a contradiction. So $\rho$ is one of $\rho_{i}$ 's, say $\rho=\rho_{s}$. But then $\sigma+\rho_{1}+\ldots+\rho_{s-1}$ is also a cone in $\operatorname{St}(\sigma)$ and its dimension is less than $\operatorname{dim}(\sigma)+(s-1)$ - a contradiction with the minimality of $\tau$. This proves the first part of the claim. The freeness of each edge $\rho_{i}$ is clear.

The lemma follows from this claim. Indeed, by definition every cone in $\operatorname{Link}(\sigma)$ is a face of a cone in $\operatorname{St}(\sigma)$. The claim implies that all cones in $\operatorname{Link}(\sigma)$ are simplicial. This proves the first assertion of the lemma. The claim also implies that for any face $\delta$ of $\sigma$ and any subset $S$ of the cones $\left\{\rho_{i}\right\}$ the cone $\delta+S$ is a face of $\tau$. The local product structure follows.

Lemma 2.13. Let $\Phi$ and $\sigma \in \Phi^{s}$ be as in the previous lemma. Choose a ray $\rho \in \sigma^{0}$ and let $\Psi \rightarrow \Phi$ be the corresponding star subdivision. If $\Phi$ is projective, so is $\Psi$.

Proof. Let $\operatorname{dim}(\sigma)=k$. Assume that $l \in \mathcal{A}^{2}(\Phi)$ is strictly convex on $\Phi$. Then $l$ is also in $\mathcal{A}^{2}(\Psi)$, but is not strictly convex on $\Psi$. Let $\tilde{l}$ be a piecewise linear function on $\Psi$ supported on $\operatorname{St}(\rho)$, such that $\left.\tilde{l}\right|_{\rho}<0$. Consider

$$
\hat{l}:=l+\epsilon \tilde{l}, \quad \text { for } 0<\epsilon<<1 .
$$

We claim that $\hat{l}$ is strictly convex on $\Psi$. Indeed, let $\delta$ and $\theta$ be two cones of dimension $n$ in $\Psi$. If one of them is not in $\operatorname{St}(\rho)$, then $\left.\hat{l}_{\delta}\right|_{\theta}<\hat{l}_{\theta}$, because the same is true for $l$ (and $\epsilon<<1$ ). So we may assume that $\delta, \theta \in \operatorname{St}(\sigma)$ and, moreover, that they are contained in the same cone 
$\tau \in \Phi$. The Claim in the proof of last lemma implies that

$$
\tau=\sigma+\sum_{i=1}^{n-k} \rho_{i}, \quad \text { where } \rho_{i} \in \operatorname{Link}(\sigma)
$$

Therefore

$$
\delta=\delta \cap \sigma+\sum_{i=1}^{n-k} \rho_{i}, \quad \theta=\theta \cap \sigma+\sum_{i=1}^{n-k} \rho_{i},
$$

and $\operatorname{dim}(\delta \cap \sigma)=\operatorname{dim}(\theta \cap \sigma)=k$. Now convexity of the boundary $\partial \sigma$ implies that $\left.\tilde{l}_{\delta}\right|_{\theta}<\tilde{l}_{\theta}$ and hence $\left.\hat{l}_{\delta}\right|_{\theta}<\hat{l}_{\theta}$, which proves the lemma.

Definition 2.14. A fan $\Delta$ is purely $n$-dimensional if every cone in $\Delta$ is contained in a cone of dimension $n$. In this case we denote by $\partial \Delta \subset \Delta$ the subfan whose support is the boundary of the support of $\Delta$. Put $\Delta^{0}=\Delta-\partial \Delta$.

Unless stated otherwise all fans are assumed to be in $V$.

\section{Review of some CONSTRUCtions And RESUlts From [BrLu] AND $[\mathrm{BBFK}]$}

1. We briefly recall the notions and results from our paper [BrLu] which are relevant to this work. All $A$-modules are graded and a morphism between $A$-modules is always homogeneous of degree 0 . Let $A^{+}$be the maximal ideal of $A$. For an $A$-module $M$ we denote by $\bar{M}=M / A^{+} M$ the corresponding (graded) $\mathbb{R}$-vector space. The shifted module $M(i)$ is defined by

$$
M(i)^{k}=M^{i+k} .
$$

Similarly we will consider shifts $H^{\bullet}(i)$ of graded vector spaces $H^{\bullet}$.

The tensor product $\otimes$ always means $\otimes_{\mathbb{R}}$.

Let $\Phi$ be a fan in $V$. We consider the (finite) set $\Phi$ as a topological space with open subsets being subfans. (In particular, $\underline{o} \in \Phi$ is the unique open point). Denote by $\operatorname{Sh}(\Phi)$ the category of sheaves of $\mathbb{R}$ vector spaces on $\Phi$.

There are two natural sheaves of rings on $\Phi$ : the constant sheaf $A_{\Phi}$ and the "structure sheaf" $\mathcal{A}_{\Phi}$. The stalk $\mathcal{A}_{\sigma}$ consists of polynomial functions on $\sigma$. These sheaves are evenly graded, $\Gamma\left(\Phi, A_{\Phi}\right)=A$, $\Gamma\left(\Phi, \mathcal{A}_{\Phi}\right)=\mathcal{A}(\Phi)$. Thus the canonical surjection of sheaves

$$
A_{\Phi} \rightarrow \mathcal{A}_{\Phi}
$$

does not induce a surjection of global sections in general.

The category of $\mathcal{A}_{\Phi}$-modules contains an important subcategory $\mathfrak{M}=$ $\mathfrak{M}(\Phi)$, consisting of flabby locally free sheaves of finite type. Namely, 
an $\mathcal{A}_{\Phi}$-module $F$ belongs to $\mathfrak{M}$ if it is a flabby sheaf and $F_{\sigma}$ is a finitely generated free $\mathcal{A}_{\Phi, \sigma}$-module for each $\sigma \in \Phi$. The category $\mathfrak{M}$ is of finite type: every object is a direct some of indecomposable ones. There exists a distinguished indecomposable object $\mathcal{L}=\mathcal{L}_{\Phi} \in \mathfrak{M}$ characterized by the property $\mathcal{L}_{\underline{o}}=\mathbb{R}$. We call $\mathcal{L}$ the minimal sheaf. It is an analogue of the equivariant intersection cohomology sheaf on the toric variety $X_{\Phi}$ corresponding to $\Phi$ (this variety exists only if the fan is rational). It is easy to see that $\mathcal{L}$ can be characterized as an object in $\mathfrak{M}$ such that $\mathcal{L}_{\underline{o}}=\mathbb{R}$ and the map

$$
\overline{\mathcal{L}_{\sigma}} \rightarrow \overline{\Gamma(\partial \sigma, \mathcal{L})}
$$

is an isomorphism for each cone $\sigma$.

The following (easy) result is the combinatorial analogue of the (equivariant) decomposition theorem for the direct image of perverse sheaves under proper maps.

Theorem 3.1. (Decomposition theorem) Let $\pi: \Psi \rightarrow \Phi$ be a subdivision. The direct image functor $\pi_{*}: \mathcal{A}_{\Psi}-\bmod \rightarrow \mathcal{A}_{\Phi}-\bmod$ takes the category $\mathfrak{M}(\Psi)$ to $\mathfrak{M}(\Phi)$. In particular the sheaf $\pi_{*} \mathcal{L}_{\Psi}$ contains $\mathcal{L}_{\Phi}$ as a (noncanonical) direct summand.

The last assertion of the theorem follows from the first one since $\left(\pi_{*} \mathcal{L}_{\Psi}\right)_{0}=\mathcal{L}_{\Psi, 0}=\mathbb{R}$.

Definition 3.2. In the notation of the last theorem a map $\alpha: \mathcal{L}_{\Phi} \rightarrow$ $\pi_{*} \mathcal{L}_{\Psi}$ is called admissible if $\alpha_{0}=i d: \mathbb{R} \rightarrow \mathbb{R}$.

Since $\mathcal{L}$ is flabby,

$$
H^{i}(\Phi, \mathcal{L})=0, \quad \text { for } i>0 .
$$

Definition 3.3 (BBFK). A fan $\Phi$ is called quasi-convex if $H^{0}(\Phi, \mathcal{L})$ is a free $A$-module.

A complete fan is quasi-convex. More precisely we have the following theorem.

Theorem 3.4 (BBFK). A quasi-convex fan is purely $n$-dimensional. A purely $n$-dimensional fan $\Phi$ is quasi-convex if and only if $|\partial \Phi|$ is a real homology manifold. In particular, $\Phi$ is quasi-convex, if $|\Phi|$ is convex.

Example 3.5. Let $\Phi$ be a complete fan and $\sigma \in \Phi$. Then the subfan $[\operatorname{St}(\sigma)]$ is quasi-convex.

For a quasi-convex fan $\Phi$ we define its intersection cohomology space as

$$
I H^{\bullet}(\Phi):=\overline{\Gamma(\Phi, \mathcal{L})}
$$


Denote by $i h_{i}(\Phi)=\operatorname{dim} I H^{i}(\Phi)$ the $i$-th Betti number of $\Phi$. If $\Phi$ is rational, then $I H(\Phi)=I H\left(X_{\Phi}\right)$.

2. Hard Lefschetz theorem. Fix a projective fan $\Phi$ with a strictly convex piecewise linear function $l$. The $A$-module $\Gamma\left(\mathcal{L}_{\Phi}\right)$ is in fact a $\mathcal{A}(\Phi)$-module; so $I H(\Phi)$ is also such. In particular $l$ induces a degree 2 endomorphism

$$
l: I H^{\bullet}(\Phi) \rightarrow I H^{\bullet+2}(\Phi) .
$$

It is known that $I H^{i}(\Phi)=0$ unless $i$ is even and $i \in[0,2 n] ; i h_{0}(\Phi)=1$, and $i h_{n-k}(\Phi)=i h_{n+k}(\Phi)$. The following "Hard Lefschetz" theorem was conjectured in $[\mathrm{BrLu}]$. It was recently proved in $[\mathrm{Ka}]$.

Theorem 3.6. (HL) For each $k>0$ the map

$$
l^{k}: I H^{n-k}(\Phi) \rightarrow I H^{n+k}(\Phi)
$$

is an isomorphism.

Corollary 3.7. For a projective fan $\Phi$ we have

$$
i h_{0}(\Phi) \leq i h_{2}(\Phi) \leq \ldots \leq i h_{2[d / 2]}(\Phi) .
$$

It was shown in $[\mathrm{BrLu}]$ that the (HL) theorem implies the Stanley conjecture on the unimodality of the generalized $h$-vector.

3. Duality. Let $\omega=\omega_{A / \mathbb{R}}=A \cdot \wedge^{n} V^{*}$ be the dualizing module for $A$. It is a free $A$-module of rank 1 generated in degree $2 n$. The dualizing sheaf on $\Phi$ is

$$
D_{\Phi}=\omega_{\underline{o}}[n],
$$

(which is a complex concentrated in degree $-n$ and supported at the origin $\underline{\underline{o}}$.)

The Verdier duality functor

$$
D: D_{c}^{b}\left(A_{\Phi}-\bmod \right)^{o p} \rightarrow D_{c}^{b}\left(A_{\Phi}-\bmod \right)
$$

is defined by

$$
D(F)=R \underline{\operatorname{Hom}}_{A_{\Phi}}\left(F, D_{\Phi}\right) .
$$

It has the following properties.

Theorem 3.8. ([BrLu]) Let $\Phi$ be a fan.

a) There is a natural isomorphism of functors $I d \rightarrow D^{2}$. So $D$ is an anti-involution of $D_{c}^{b}\left(A_{\Phi}-\bmod \right)$.

b) $D(\mathfrak{M}(\Phi))=\mathfrak{M}(\Phi)$.

c) $D \mathcal{L} \simeq \mathcal{L}$.

The last assertion of the theorem implies the Poincaré duality for $I H(\Phi)$ for a complete fan $\Phi$.

For a deeper study of our duality functor we will need the notion of a cellular complex which we recall next. 
4. Cellular complex $C^{\bullet}(\cdot)$. Choose an orientation of each (nonzero) cone in the fan $\Phi$. Then to each sheaf $F$ on $\Phi$ we can associate its cellular complex

$$
C^{0}(F) \stackrel{\partial}{\rightarrow} C^{1}(F) \stackrel{\partial}{\rightarrow} \ldots \stackrel{\partial}{\rightarrow} C^{n}(F)
$$

where

$$
C^{i}(F)=\bigoplus_{\operatorname{dim}(\sigma)=n-i} F_{\sigma},
$$

and the differential $\partial$ is the sum of the restriction maps $F_{\sigma} \rightarrow F_{\tau}$ with plus or minus sign depending on whether the orientations of $\sigma$ and $\tau$ agree or not. Sometimes we will be more specific and write $C_{\Phi}^{\bullet}(F)$ for $C^{\bullet}(F)$. Note that $C^{\bullet}(\cdot)$ is an exact functor from sheaves on $\Phi$ to complexes.

In particular, we get a functor

$$
C^{\bullet}(\cdot): D^{b}\left(A_{\Phi}-\bmod \right) \rightarrow D^{b}(A-\bmod ) .
$$

Note that $C^{\bullet}\left(D_{\Phi}\right)=\omega($ a complex concentrated in degree 0$)$.

The following proposition will be used extensively in this work.

Proposition $3.9(\mathrm{BrLu})$. For any fan $\Phi$ there exists a natural isomorphism of functors $D_{c}^{b}\left(A_{\Phi}-\bmod \right)^{o p} \rightarrow D_{c}^{b}(A-\bmod )$

$$
\mathbb{R} \Gamma(\Phi, D(\cdot))=\mathbb{R} \operatorname{Hom}_{A_{\Phi}}^{\bullet}\left(\cdot, D_{\Phi}\right) \rightarrow \mathbb{R} \operatorname{Hom}_{A}^{\bullet}\left(C^{\bullet}(\cdot), \omega\right),
$$

induced by the functor $C^{\bullet}(\cdot)$.

This proposition shows that the Verdier duality can be considered as the Borel-Moore duality (see $[\mathrm{BrLu}])$. Namely, for $F \in D_{c}^{b}\left(A_{\Phi}-\bmod \right)$ the assignment

$$
\sigma \mapsto C^{\bullet}\left(F_{[\sigma]}\right)
$$

defines the co-sheaf (of complexes) of sections of $F$ "with compact supports". (Here $F_{[\sigma]}$ denotes the extension by zero of the restriction of $F$ to $[\sigma])$. It follows from the above proposition that

$$
\mathbb{R} \Gamma([\sigma], D(F))=\mathbb{R} \operatorname{Hom}_{A}^{\bullet}\left(C^{\bullet}\left(F_{[\sigma]}\right), \omega\right),
$$

where the right hand side is the analogue of the topological Borel-Moore duality.

The cohomology groups $H^{i}\left(C^{\bullet}(F)\right)$ tend to be related to the cohomology groups $H^{i}(\Phi, F)$. For example, if $\Phi$ is complete and the orientations of all $n$-dimensional cones agree, then the natural map $\Gamma(\Phi, F) \rightarrow C^{0}(F)$ induces an isomorphism $\Gamma(F) \simeq H^{0}\left(C^{\bullet}(F)\right)$. Moreover, the other cohomology groups also coincide, i.e. we have a canonical functorial quasi-isomorphism of complexes (for a complete fan $\Phi$ )

$$
\mathbb{R} \Gamma(\Phi, F) \simeq C^{\bullet}(F)
$$


$([\mathrm{BrLu}])$. This shows that the cellular complex makes sense once we choose a global orientation on $V$. In fact for our purposes it will be necessary to choose a volume form on $V$. So from now on we make the following assumption.

Assumption 1. We assume that a nonzero element $\Omega=\Omega_{V} \in \wedge^{n} V^{*}$ has been fixed.

This choice determines a trivialization $\omega=A(2 n)$. Thus in particular we obtain an isomorphism $C^{\bullet}\left(D_{\Phi}\right)=C^{n}\left(D_{\Phi}\right)=A(2 n)$. We want to see how this isomorphism changes in a natural way once we make a different choice of the volume form. For this assume, for simplicity, that $\Phi$ is complete.

Two different choices of orientations of cones in $\Phi$ produce two functors $C_{1}^{\bullet}$ and $C_{2}^{\bullet}$ and there exists an isomorphism $\phi: C_{1}^{\bullet} \rightarrow C_{2}^{\bullet}$. We want $\phi$ to be compatible with the canonical isomorphisms $H^{0}\left(C_{1}^{\bullet}(F)\right)=$ $\Gamma(F)=H^{0}\left(C_{2}^{\bullet}(F)\right)$. This forces $\phi^{0}: C_{1}^{0} \rightarrow C_{2}^{0}$ to be the identity map, and hence defines uniquely the isomorphisms $\phi^{i}: C_{1}^{i} \rightarrow C_{2}^{i}$. For example, if the two choices of orientations are the same for all cones of dimension $<n$, and are opposite on the maximal cones (i.e. only the global orientation is changed), then $\phi^{i}=-1$ for all $i>0$. One can easily check that the sign of $\phi^{n}$ depends only on the comparison of the orientations of maximal cones, i.e. $\phi^{n}=1$ if the global orientations agree and $\phi^{n}=-1$ if they are opposite.

In particular, let $W$ be a vector space and $W_{\underline{o}}$ be the sheaf on $\Phi$, which is equal to $W$ on $\underline{o}$ and zero elsewhere. Choose a cellular complex $C^{\bullet}$ and get an isomorphism $C^{\bullet}\left(W_{\underline{o}}\right)=C^{n}\left(W_{\underline{o}}\right) \simeq W[-n]$. However, as was explained above if we choose a different cellular complex with the opposite global orientation, then the above isomorphism changes sign.

A certain "cancellation" of two ambiguities occurs in the previous example if $W=\omega$. Indeed, our choice of the volume form $\Omega$ defines an isomorphism $\omega=A(2 n)$ and also an isomorphism $C^{\bullet}\left(D_{\Phi}\right)=\omega$. So we get an isomorphism

$$
C^{\bullet}\left(D_{\Phi}\right)=A(2 n)
$$

But the arguments above show that the volume form $-\Omega$ defines the same (!) isomorphism. Hence this last isomorphism is independent of $\Omega$ up to multiplication by a positive number.

Remark 3.10. The above situation is similar to the fact that on a smooth orientable connected manifold the top cohomology group with coefficients in the orientation sheaf is canonically isomorphic to $\mathbb{Z}$.

Let us apply Proposition 3.9 together with the last argument to our main example of the sheaf $\mathcal{L}$. 
Theorem 3.11. Assume that the fan $\Phi$ is complete. Then the volume form $\Omega$ defines a canonical isomorphism of $A$-modules

$$
\Gamma(D \mathcal{L})=\operatorname{Hom}_{A}(\Gamma(\mathcal{L}), A(2 n))
$$

A different choice of a volume form defines the same isomorphism up to multiplication by a positive real number.

Proof. Indeed, the complex $C^{\bullet}(\mathcal{L})$ is acyclic except in degree 0 , and $H^{0}\left(C^{\bullet}(\mathcal{L})\right)=\Gamma(\mathcal{L})$ is a free $A$-module.

Remark 3.12. Note that both $\mathcal{L}$ and $D \mathcal{L}$ are $\mathcal{A}_{\Phi^{-}}$-modules (not just $A_{\Phi}$-modules). The $\mathcal{A}(\Phi)$-module structure on $\Gamma(D \mathcal{L})$ comes from such structure on $\Gamma(\mathcal{L})$ via the isomorphism of the last theorem.

5. The Hodge-Riemann bilinear relations. In order to formulate the second main theorem we need to make the following assumption.

Assumption 2. From now on we assume that the (HL) theorem holds for all complete fans of dimension $<n$.

Lemma 3.13. Let $\Phi$ be a fan with the minimal sheaf $\mathcal{L}_{\Phi}$. Then for any cone $\tau \in \Phi$ of dimension $k \geq 1$ the following hold:

1) The stalk $\mathcal{L}_{\Phi, \tau}$ is generated (as the $A$-module) in degrees $<k$.

2) The costalk $\Gamma_{\{\tau\}} \mathcal{L}_{\Phi}:=\operatorname{Ker}\left(\mathcal{L}_{\Phi, \tau} \rightarrow \Gamma\left(\partial \tau, \mathcal{L}_{\Phi}\right)\right)$ is generated in degrees $>k$.

Proof. To simplify the notation assume that $k=n$. Choose a ray $\rho$ in the interior of $\tau$ and consider the projection $p: V \rightarrow \bar{V} \simeq \mathbb{R}^{n-1}$. Then $\overline{\partial \tau}:=p(\partial \tau)$ is a complete fan in $\bar{V}$ and $\partial \tau \subset V$ is a graph of a stictly convex piecewise linear function $\bar{l}$ on $\overline{\partial \tau}$. Let $A_{\bar{V}}$ be the algebra of polynomial functions on $\bar{V}$. Note then $A=A_{\bar{V}}[\bar{l}]$. The projection $p: \partial \tau \rightarrow \overline{\partial \tau}$ defines an isomorphism of $A_{\bar{V}}$ - modules

$$
\Gamma\left(\partial \tau, \mathcal{L}_{\Phi}\right) \simeq \Gamma\left(\overline{\partial \tau}, \mathcal{L}_{\overline{\partial \tau}}\right) .
$$

So in particular $\Gamma\left(\partial \tau, \mathcal{L}_{\Phi}\right)$ is a free $A_{\bar{V}}$-module. By our Assumption 2 above $\bar{l}$ acts on $I H(\overline{\partial \tau})$ as a Lefschetz operator. This implies both assertion of the lemma because the residue map

$$
\overline{\mathcal{L}_{\Phi, \tau}} \rightarrow \overline{\Gamma\left(\partial \tau, \mathcal{L}_{\Phi}\right)}
$$

is an isomorphism.

Corollary 3.14. $\operatorname{End}_{A_{\Phi}}\left(\mathcal{L}_{\Phi}\right)=\mathbb{R}$. 
Proof. This follows from the previous lemma and from the fact that $\mathcal{L}_{\Phi, \underline{o}}=\mathbb{R}$ by induction on the dimension of cones in $\Phi$. Indeed, let $\sigma \in \Phi$ be of dimension $d$ and consider the exact sequence of $A$-modules

$$
0 \rightarrow \Gamma_{\{\sigma\}} \mathcal{L}_{\Phi} \rightarrow \mathcal{L}_{\Phi, \sigma} \rightarrow \Gamma\left(\partial \sigma, \mathcal{L}_{\Phi}\right) \rightarrow 0 .
$$

Since $\mathcal{L}_{\Phi, \sigma}\left(\right.$ resp. $\left.\Gamma_{\{\sigma\}} \mathcal{L}_{\Phi}\right)$ is generated in degrees $<d$ (resp. $>d$ ) the identity endomorphism of $\mathcal{L}_{[\partial \sigma]}$ can be extended to an endomorphism of $\mathcal{L}_{[\sigma]}$ in a unique way.

Lemma 3.15. There exists a canonical isomorphism of $\mathcal{A}_{\Phi}$-modules

$$
\epsilon_{\Phi}: \mathcal{L}_{\Phi} \rightarrow D\left(\mathcal{L}_{\Phi}\right)
$$

Proof. Since the sheaves $\mathcal{L}_{\Phi}$ and $D\left(\mathcal{L}_{\Phi}\right)$ are isomorphic, by the previous corollary it suffices to find a canonical isomorphism of the stalks $\mathcal{L}_{\Phi, \underline{o}}$ and $D\left(\mathcal{L}_{\Phi}\right)_{\underline{o}}$. But

$$
D\left(\mathcal{L}_{\Phi}\right)_{\underline{o}}=R \operatorname{Hom}_{A}\left(\mathcal{L}_{\Phi, \underline{o}}, D_{\Phi, \underline{o}}\right)=R \operatorname{Hom}_{A}(\mathbb{R}, \omega[n])=\operatorname{Ext}_{A}^{n}(\mathbb{R}, \omega[n]) .
$$

To compute the last Ext group take the canonical Koszul resolution of the $A$-module $\mathbb{R}$

$$
0 \rightarrow \omega \rightarrow A \otimes \wedge^{n-1} V^{*} \rightarrow \ldots \rightarrow A \otimes V^{*} \rightarrow A \rightarrow \mathbb{R} .
$$

Then $\operatorname{Ext}_{A}^{n}(\mathbb{R}, \omega[n])=\operatorname{Hom}_{A}(\omega[n], \omega[n])=\mathbb{R}$.

Corollary 3.16. Assume that the fan $\Phi$ is complete. The volume form $\Omega$ defines a canonical nondegenerate pairing

$$
[\cdot, \cdot]=[\cdot, \cdot]_{\Phi}=\Gamma\left(\mathcal{L}_{\Phi}\right) \times \Gamma\left(\mathcal{L}_{\Phi}\right) \rightarrow A(2 d),
$$

and hence a canonical nondegenerate pairing

$$
(\cdot, \cdot)=(\cdot, \cdot)_{\Phi}: I H(\Phi) \times I H(\Phi) \rightarrow \mathbb{R}(2 d) .
$$

A different volume form defines the same pairings up to multiplication by a positive real number. These pairings are $\mathcal{A}(\Phi)$-bilinear.

Proof. This follows from the Theorem 3.11, Remark 3.12 and Lemma 3.15 .

In section 5 below we will construct a similar canonical pairing for quasi-convex fans.

The next theorem is the analogue of the Hodge-Riemann bilinear relations on the primitive intersection cohomology of complex algebraic varieties.

Theorem 3.17. (HR) Let $\Phi$ be a projective fan with a strictly convex piecewise linear function $l$. For $k \geq 0$ put

$$
\operatorname{Prim}_{l} I H^{n-k}(\Phi):=\left\{b \in I H^{n-k} \mid l^{k+1} b=0\right\} .
$$


Then the quadratic form $Q_{l}(a)=(-1)^{\frac{n-k}{2}}\left(a, l^{k} a\right)_{\Phi}$ is positive definite on $\operatorname{Prim}_{l} I H^{n-k}(\Phi)$.

6. Intersection cohomology of pl-isomorphic fans and of $\operatorname{St}[\sigma]$. Let $\Phi, \Psi$ be fans and $\gamma:|\Phi| \rightarrow|\Psi|$ be a pl-isomorphism. Then there exists an isomorphism of sheaves $\gamma^{*} \mathcal{A}_{\Psi} \simeq \mathcal{A}_{\Phi}$. Hence also an isomorphism of minimal sheaves $\gamma^{*} \mathcal{L}_{\Psi} \simeq \mathcal{L}_{\Phi}$ and of the global sections $\Gamma\left(\Phi, \mathcal{L}_{\Phi}\right) \simeq \Gamma\left(\Psi, \mathcal{L}_{\Psi}\right)$. This last isomorphism however does not preserve the structure of a module over the global polynomial functions, unless $\gamma$ is induced by a linear isomorphism of the ambient vector spaces. Thus, for example, if the pl-isomorphic fans are quasi-convex, there is no canonical isomorphism between $I H(\Phi)$ and $I H(\Psi)$. Note however, that these graded vector spaces are isomorphic, since the graded spaces $\Gamma\left(\mathcal{L}_{\Phi}\right)$ and $\Gamma\left(\mathcal{L}_{\Psi}\right)$ have the same Hilbert function.

Assume that a complete fan $\Phi$ has a local product structure at a cone $\sigma$. Let $p: V \rightarrow \bar{V}=V /\langle\sigma\rangle$ be the projection, $\Phi_{\sigma}=p(\operatorname{Link}(\sigma))-$ the complete fan in $\bar{V}$. Note that the projection $p$ makes $\Gamma\left([\operatorname{St}(\sigma)], \mathcal{L}_{\Phi}\right)$ an $\mathcal{A}\left(\Phi_{\sigma}\right)$-module. Indeed, by the local product structure at $\sigma$ the projection $p(\tau)$ of any cone $\tau \in[\operatorname{St}(\sigma)]$ is a cone in $\Phi_{\sigma}$. In particular this makes $I H([\operatorname{St}(\sigma)])$ a $\mathcal{A}\left(\Phi_{\sigma}\right)$-module.

Lemma 3.18. There exist natural isomorphisms of $\mathcal{A}\left(\Phi_{\sigma}\right)$-modules

$$
\begin{aligned}
\Gamma\left([\operatorname{St}(\sigma)], \mathcal{L}_{\Phi}\right) & \simeq \Gamma\left(\Phi_{\sigma}, \mathcal{L}_{\Phi}\right) \otimes \mathcal{L}_{\Phi, \sigma} \\
I H([\operatorname{St}(\sigma)]) & \simeq I H\left(\Phi_{\sigma}\right) \otimes \overline{\mathcal{L}_{\Phi, \sigma}}
\end{aligned}
$$

with the trivial $\mathcal{A}\left(\Phi_{\sigma}\right)$-module structure on $\mathcal{L}_{\Phi, \sigma}$. In particular, if $\sigma$ is simplicial, i.e. $\mathcal{L}_{\Phi, \sigma}=\mathcal{A}_{\Phi, \sigma}$, then

$$
I H([\operatorname{St}(\sigma)])=I H\left(\Phi_{\rho}\right) \text {. }
$$

Proof. Let $A_{\sigma}$ be the polynomial functions on $\sigma$. Choose a projection $V \rightarrow\langle\sigma\rangle$ and identify $A_{\sigma}$ as a subalgebra of $A$.

Let $p_{\sigma}: \operatorname{St}(\sigma) \rightarrow \Phi_{\sigma}$ denote the restriction of the projection $\mathrm{p}$ to $\operatorname{St}(\sigma)$. The sheaf $A_{\sigma} \otimes p_{\sigma}^{-1} \mathcal{L}_{\Phi_{\sigma}}$ extended by zero to $\Phi$ satisfies the definition of a minimal sheaf based at $\sigma$ in [BrLu] (5.1). (It is denoted by $\mathcal{L}_{\Phi}^{\sigma}$ in $[\mathrm{BrLu}]$.) Note that the restriction $\left.\mathcal{L}_{\Phi}\right|_{\mathrm{St}(\sigma)}$ extended by zero to $\Phi$ is also a minimal sheaf based at $\sigma$. By Proposition 5.2 in [BrLu] $\left.\mathcal{L}_{\Phi}\right|_{\operatorname{St}(\sigma)} \simeq \mathcal{L}_{\Phi}^{\sigma} \otimes \overline{\mathcal{L}_{\Phi, \sigma}}$. Therefore

$$
\Gamma\left(\operatorname{St}(\sigma), \mathcal{L}_{\Phi}\right) \simeq \Gamma\left(\Phi_{\sigma}, \mathcal{L}_{\Phi_{\sigma}}\right) \otimes A_{\sigma} \otimes \overline{\mathcal{L}_{\Phi, \sigma}}=\Gamma\left(\Phi_{\sigma}, \mathcal{L}_{\Phi_{\sigma}}\right) \otimes \mathcal{L}_{\Phi, \sigma} .
$$

This is an isomorphism of $\mathcal{A}\left(\Phi_{\sigma}\right)$-modules. It remains to note that

$$
\Gamma\left([\operatorname{St}(\sigma)], \mathcal{L}_{\Phi}\right)=\Gamma\left(\operatorname{St}(\sigma), \mathcal{L}_{\Phi}\right) .
$$




\section{ReVieW OF The "SMOOTH" CASE: Simple POLYTOPES AND SIMPLICIAL FANS}

1. Review of Timorin's work on simple polytopes. The analogue of Hodge-Riemann bilinear relations for simple polytopes was proved by McMullen in $[\mathrm{McM}]$. Later Timorin gave a simpler proof in [Ti]. Here we recall his main results.

Consider the dual space $W=V^{*}$. Let $P \subset W$ be a convex polytope of dimension $n$. We assume that $P$ is simple, i.e. at each vertex of $P$ there meet exactly $n$ faces of dimension $n-1$.

Definition 4.1. Two convex polytopes $P^{\prime}, P^{\prime \prime} \subset W$ are called analogous if their hyperplane faces can be pairwise matched so that the matched faces

1) have the same outward normal direction,

2) are analogous.

Any two segments on a line are analogous.

Clearly being analogous is an equivalence relation. Let $\mathcal{P}^{+}(P)$ denote the collection of all polytopes in $W$ analogous to $P$. If $P^{\prime}, P^{\prime \prime} \in \mathcal{P}^{+}(P)$, then their Minkowski sum

$$
P^{\prime}+P^{\prime \prime}=\left\{p^{\prime}+p^{\prime \prime} \mid p^{\prime} \in P^{\prime}, p^{\prime \prime} \in P^{\prime \prime}\right\}
$$

is also in $\mathcal{P}^{+}(P)$. Also

$$
\lambda P^{\prime}=\left\{\lambda p^{\prime} \mid p^{\prime} \in P^{\prime}\right\}, \quad \lambda>0
$$

is in $\mathcal{P}^{+}(P)$. Thus $\mathcal{P}^{+}(P)$ has a structure of a convex cone. It can be complemented to a vector space $\mathcal{P}(P)$ by considering formal differences of polytopes.

Choose a volume form $\Omega_{W} \in \wedge^{n} V$ on $W$ to be the dual of $\Omega_{V}$ and orient $W$ in such a way that

$$
\operatorname{Vol}(P):=\int_{P} \Omega_{W}>0 .
$$

Proposition $4.2(\mathrm{Ti})$. The volume function $V$ ol defined on $\mathcal{P}^{+}(P)$ extends to a homogeneous polynomial function of degree $n$ on $\mathcal{P}(P)$. $W e$ denote this extended function again by $\mathrm{Vol}$.

Let $P_{1}, \ldots, P_{m}$ be the faces of $P$ of dimension $n-1$. Choose the corresponding outward normal covectors $\xi_{1}, \ldots, \xi_{m} \in V$. That is, each $\left.\xi_{i}\right|_{P}$ achieves its maximum exactly on $P_{i}$. Then we associate to $P$ the numbers $H_{1}, \ldots, H_{m}$ defined by

$$
H_{i}:=\max _{p \in P}\left\{\xi_{i}(p)\right\}=\xi_{i}\left(P_{i}\right) .
$$


The functions $H_{1}, \ldots, H_{m}$ form a system of linear coordinates on $\mathcal{P}(P)$.

2. The polytope algebra $A(P)$. Let Diff be the algebra of differential operators with constant coefficients on $\mathcal{P}(P)$. It is a commutative polynomial ring with generators

$$
\partial_{i}=\frac{\partial}{\partial H_{i}}, \quad i=1, \ldots, m .
$$

Let $I \subset \operatorname{Diff}$ be the ideal

$$
I=\{d \in \operatorname{Diff} \mid d V o l=0\} .
$$

Put $A(P):=\operatorname{Diff} / I$. Since $V o l$ is a homogeneous polynomial, the ideal $I$ is graded and so is the ring $A(P)$ :

$$
A(P)=\oplus_{k=0}^{n} A_{k}(P) .
$$

The following proposition is almost obvious.

Proposition $4.3(\mathrm{Ti})$. The formula $(\alpha, \beta)_{T}=\alpha \beta$ Vol defines a nondegenerate pairing

$$
(\cdot, \cdot)_{T}: A_{k}(P) \otimes A_{n-k}(P) \rightarrow \mathbb{R} .
$$

Corollary 4.4. $\operatorname{dim} A_{k}=\operatorname{dim} A_{n-k}$.

Theorem $4.5(\mathrm{Ti})$. We have $\operatorname{dim} A_{k}=h_{k}(P)$ - the $k$-th component of the h-vector $h(P)$.

3. Presentation of the algebra $A(P)$. We have a natural embedding of linear spaces

$$
i: W \hookrightarrow D i f f, \quad i(a)=\sum_{i=1}^{m} \xi_{i}(a) \partial_{i} .
$$

Let $P_{i_{1}}, \ldots, P_{i_{k}}$ be different faces of $P$ of dimension $n-1$. Put

$$
D_{i_{1} \ldots i_{k}}:=\partial_{i_{1}} \ldots \partial_{i_{k}} \in \text { Diff. }
$$

Theorem $4.6(\mathrm{Ti})$. 1) For all $a \in W, i(a) \in I$ (translation invariance of the volume function).

2) If $P_{i_{1}} \cap \ldots \cap P_{i_{k}}=\emptyset$, then $D_{i_{1} \ldots i_{k}} \in I$.

3) The ideal $I$ is generated by elements $i(a), D_{i_{1} \ldots i_{k}}$ as in 1), 2). 
4. A basis for $A(P)$. Fix a general linear function $t$ on $W$. For each vertex $p \in P$ let $i n d e x(p)$ be the number of edges coming out of $p$ on which the maximum of $t$ is attained at $p$. That is index $(p)$ is the number of edges which go down from $p$. Consider the face $F(p)$ spanned by all these edges. We have $\operatorname{dim} F(p)=i n \operatorname{dex}(p)$. Thus we associated one face to each vertex (the interior of $P$ is associated to the "highest" vertex).

Note that each face $F \subset P$ of codimension $k$ is the intersection of exactly $k$ hyperplane faces: $F=P_{i_{1}} \cap \ldots \cap P_{i_{k}}$. Put $D_{F}:=\partial_{i_{1}} \ldots \partial_{i_{k}} \in$ Diff.

Theorem $4.7(\mathrm{Ti})$. The monomials $\left\{D_{F(p)} \mid p\right.$ is a vertex in $\left.P\right\}$ form a basis of $A(P)$. More precisely, monomials $\left\{D_{F(p)} \mid\right.$ index $\left.(p)=k\right\}$ form a basis of $A_{n-k}$.

5. The Lefschetz operator. Consider the operator

$$
L=L_{P}=\sum_{i=1}^{m} H_{i}(P) \partial_{i} \in A_{1}(P) .
$$

Theorem 4.8 (Ti). The operator (of multiplication by) L acts as a Lefschetz operator on $A(P)$, i.e. for all $i \leq n / 2$ the map

$$
L^{n-2 i}: A_{i}(P) \rightarrow A_{n-i}(P)
$$

is an isomorphism.

Theorem $4.9(\mathrm{Ti})$. Let

$$
\operatorname{Prim}_{i}(P)=\left\{a \in A_{i}(P) \mid L^{n-2 i+1} a=0\right\}
$$

be the primitive part of $A_{i}(P)$. Then the symmetric bilinear form $\langle a, b\rangle_{i}=(-1)^{i}\left(a, L^{n-2 i} b\right)_{T}$ is positive definite on $\operatorname{Prim} A_{i}(P)$.

Theorem 4.10 (Ti). We have $L_{P}^{n} V o l=n ! \operatorname{Vol}(P)$, i.e. $\left(L_{P}^{n}, 1\right)_{T}=$ $n ! \operatorname{Vol}(P)$.

6. The dual picture in terms of fans after Brion [Bri]. Let $P \subset$ $W$ be a simple polytope and $\Phi=\Phi_{P} \subset V$ be its outer normal fan (Example 2.2). Since $\Phi$ is simplicial, $\mathcal{L}_{\Phi}=\mathcal{A}_{\Phi}$ and we denote

$$
H^{\bullet}(\Phi)=I H^{\bullet}(\Phi) \text {. }
$$

Then $H^{\bullet}(\Phi)$ is a (evenly) graded algebra

$$
H^{\bullet}(\Phi)=\oplus_{k=0}^{n} H^{2 k}(\Phi) .
$$

Proposition 4.11 (Bri). $\operatorname{dim} H^{2 k}(\Phi)=h_{k}(P)$. 
7. A pairing $(\cdot, \cdot)_{B}$ on $H^{\bullet}(\Phi)$. Brion defines a (degree zero homogeneous) map of $A$-modules $\zeta: \mathcal{A}(\Phi) \rightarrow A(2 n)$. It follows from the last proposition that such a map is unique up to a scalar factor. Let us recall the construction. For each $n$-dimensional cone $\sigma$, denote by $\mathcal{F}_{\sigma}$ the product of equations of the facets of $\sigma$. Then $\mathcal{F}_{\sigma} \in A$ is uniquely defined up to scalar multiplication. One normalizes $\mathcal{F}_{\sigma}$ as follows: the equations of the facets are nonnegative on $\sigma$ and their wedge product is equal $\pm \Omega_{V}$. Denote by $\phi_{\sigma} \in \mathcal{A}(\Phi)$ the function such that

$$
\phi_{\sigma}(v)= \begin{cases}\mathcal{F}_{\sigma}(v) & \text { if } v \in \sigma \\ 0, & \text { otherwise }\end{cases}
$$

Thus $\phi_{\sigma} \in \mathcal{A}_{2 n}(\Phi)$ and it vanishes outside $\sigma$.

For $f \in \mathcal{A}(\Phi)$ define

$$
\zeta(f):=\sum_{\operatorname{dim}(\sigma)=n} \frac{f_{\sigma}}{\mathcal{F}_{\sigma}} .
$$

Theorem 4.12 (Bri). The map $\zeta$ is a well defined map $\zeta: \mathcal{A}(\Phi) \rightarrow$ $A(2 n)$ such that

1) $\zeta$ is A-linear;

2) $\zeta\left(\phi_{\sigma}\right)=1$ for all $n$-dimensional $\sigma \in \Phi$.

Clearly, $\zeta(f)=0$ if $\operatorname{deg}(f)<2 n$.

The map $\zeta$ induces a symmetric pairing

$$
[\cdot, \cdot]_{B}: \mathcal{A}(\Phi) \times \mathcal{A}(\Phi) \rightarrow A(2 n), \quad[a, b]_{B}=\zeta(a b) .
$$

We also obtain a nonzero linear function

$$
\bar{\zeta}: H^{2 n}(\Phi) \rightarrow A / A^{+}=\mathbb{R}(2 n),
$$

and hence a pairing

$$
(\cdot, \cdot)_{B}: H^{\bullet}(\Phi) \times H^{\bullet}(\Phi) \rightarrow \mathbb{R}(2 n), \quad(x, y)_{B}=\bar{\zeta}(x y)
$$

Proposition 4.13 (Bri). The pairings $[\cdot, \cdot]_{B}$ and $(\cdot, \cdot)_{B}$ are nondegenerate.

Remark 4.14. Notice that if the form $\Omega_{V}$ is changed by a factor of $r \in \mathbb{R}$, then the map $\zeta$ and hence the pairings $[\cdot, \cdot]_{B},(\cdot, \cdot)_{B}$ are changed by the factor $|r|^{-1}$.

Recall the support function $H_{P} \in \mathcal{A}(\Phi)$ of $P$ (Example 2.2):

$$
H_{P}(v)=\max _{x \in P}\langle x, v\rangle \text {. }
$$

Note, that if $P$ contains the origin in $W$, then $H_{P}$ is nonnegative.

Theorem 4.15 (Bri). We have $\zeta\left(H_{P}^{n}\right)=n ! \operatorname{Vol}(P)$. 


\section{Relation between the pictures of Timorin and Brion.}

Theorem 4.16. There exists a natural isomorphism of algebras

$$
\beta: A(P) \rightarrow H(\Phi), \quad \beta: A_{k}(P) \stackrel{\sim}{\rightarrow} H^{2 k}(\Phi),
$$

such that $\beta\left(L_{P}\right)=H_{P}$, and $(a, b)_{T}=(\beta(a), \beta(b))_{B}$.

Proof. First note that there is a natural homomorphism of algebras $\tilde{\beta}: \operatorname{Diff} \rightarrow \mathcal{A}(\Phi)$. Indeed, vectors in $V$ which lie in the 1-dimensional cones in $\Phi$ are naturally linear functions on the space $\mathcal{P}(P)$. Hence a differential operator of order 1 on $\mathcal{P}(P)$ defines a linear function on each 1-dimensional cone of $\Phi$. However, since $\Phi$ is simplicial, every such function extends uniquely to a piecewise linear function on $\Phi$. This defines the homomorphism $\tilde{\beta}: \operatorname{Diff} \rightarrow \mathcal{A}(\Phi)$.

Notice that the generators $D_{i_{1}, \ldots, i_{k}}$ of the ideal $I \subset \operatorname{Diff}$ lie in the kernel of $\tilde{\beta}$. Indeed, let $P_{i}$ be a face of $P$ of dimension $n-1, \xi_{i} \in V-$ a corresponding outward normal covector, $\rho_{i} \in \Phi$ - the corresponding 1-dimensional cone in $\Phi$ (so $\xi_{i} \in \rho_{i}$ ), $\partial_{i} \in \operatorname{Diff}$ - the corresponding derivation. Then the piecewise linear function $\tilde{\beta}\left(\partial_{i}\right)$ takes value 1 on $\xi_{i}$ and is zero on all other 1-dimensional cones. Thus $\tilde{\beta}\left(\partial_{i}\right)$ is nonzero only on the star of $\rho_{i}$. Now, it is clear that if the intersection of $n-1$ dimensional faces $P_{i_{1}}, \ldots, P_{i_{k}}$ is empty, then so is the intersection of the stars of the corresponding 1-dimensional cones $\rho_{i_{1}}, \ldots, \rho_{i_{k}}$. Hence

$$
\tilde{\beta}\left(D_{i_{1}, \ldots i_{k}}\right)=\tilde{\beta}\left(\partial_{i_{1}} \cdot \ldots \cdot \partial_{i_{k}}\right)=0 .
$$

Notice also that $\tilde{\beta}$ maps the subspace $i(W) \subset \operatorname{Diff}$ to the space of linear functions on $V$.

It follows from Theorem 4.6 that $\tilde{\beta}$ descends to a ring homomorphism $\beta: A(P) \rightarrow H(\Phi)$. This homomorphism is surjective, since $\tilde{\beta}$ is surjective (functions $\tilde{\beta}\left(\partial_{i}\right)$ as above generate $\mathcal{A}(\Phi)$ ). Since $\operatorname{dim} A(P)=$ $\operatorname{dim} H(\Phi), \beta$ is an isomorphism.

It is clear that $\beta\left(L_{P}\right)=H_{P}$ and the last assertion follows from Theorems 4.10 and 4.15 above.

9. Hard Lefschetz and Hodge-Riemann bilinear form for simplicial fans. Let $\Phi$ be a complete simplicial fan in $V$ with a strictly convex piecewise linear function $l$. Then there exists a convex polytope $P \subset W$ such that $\Phi=\Phi_{P}$ and $l=H_{P}$.

The following theorems are immediate corollaries of Theorem 4.8, 4.9 and 4.16 above.

Theorem 4.17. For $\Phi$ and $l$ as above the multiplication by $l$ acts as a Lefschetz operator on $H(\Phi)$, i.e.

$$
l^{k}: H^{n-k}(\Phi) \stackrel{\sim}{\rightarrow} H^{n+k}(\Phi)
$$


for all $k \geq 1$.

Theorem 4.18. Let $\Phi, l$ be as in the last theorem. For $k \geq 1$ put

$$
\operatorname{Prim} H^{n-k}(\Phi):=\left\{b \in H^{n-k}(\Phi) \mid l^{k+1} b=0\right\} .
$$

Then the symmetric bilinear form $(-1)^{\frac{n-k}{2}}(a, b)_{B}$ is positive definite on $\operatorname{Prim} H^{n-k}(\Phi)$.

\section{DUAlity FOR QUASI-CONVEX FANS}

1. Following the idea in $[\mathrm{BBFK}]$ we develop the duality for quasiconvex fans. Recall that a fan $\Delta$ in $V$ is quasi-convex if $\Gamma\left(\Delta, \mathcal{L}_{\Delta}\right)=$ $\Gamma(\mathcal{L})$ is a free $A$-module.

Let $\Delta$ be a quasi-convex fan in $V, \Delta^{0}:=\Delta-\partial \Delta$. Recall that $\partial \Delta$ and $\Delta^{0}$ are open and closed subsets of $\Delta$ respectively. For a sheaf $F$ on $\Delta$ we denote by $\Gamma_{\Delta^{0}} F$ the global sections of $F$ supported on $\Delta^{0}$. That is

$$
\Gamma_{\Delta^{0}} F=\operatorname{Ker}\{\Gamma(\Delta, F) \rightarrow \Gamma(\partial \Delta, F)\} .
$$

Consider the standard short exact sequence of sheaves

$$
0 \rightarrow \mathcal{L}_{\partial \Delta} \rightarrow \mathcal{L} \rightarrow \mathcal{L}_{\Delta^{0}} \rightarrow 0
$$

and the induced short exact sequence of cellular complexes

$$
0 \rightarrow C^{\bullet}\left(\mathcal{L}_{\partial \Delta}\right) \rightarrow C^{\bullet}(\mathcal{L}) \rightarrow C^{\bullet}\left(\mathcal{L}_{\Delta^{0}}\right) \rightarrow 0
$$

It is proved in [BBFK] (Thms 4.3 and 4.11) that in the natural commutative diagram

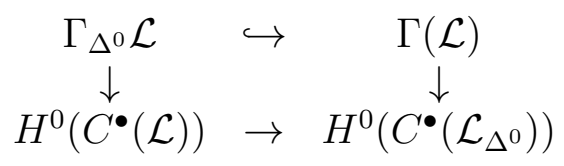

the vertical maps are isomorphisms. Moreover, the complexes $C^{\bullet}(\mathcal{L})$ and $C^{\bullet}\left(\mathcal{L}_{\Delta^{0}}\right)$ are acyclic in degrees other than zero.

Recall that $I H^{\bullet}(\Delta):=\overline{\Gamma(\mathcal{L})}$. One also defines

$$
I H^{\bullet}(\Delta, \partial \Delta):=\overline{\Gamma_{\Delta^{0}} \mathcal{L}}
$$

It was shown in $[\mathrm{BBFK}]$ that there exists a (noncanonical) duality between the free $A$-modules $\Gamma(\mathcal{L})$ and $\Gamma_{\Delta^{0}}$ and hence a duality between the graded vector spaces $I H(\Delta)$ and $I H(\Delta, \partial \Delta)$. We are going to make this duality canonical and to show that it is compatible with various natural constructions, such as embeddings of fans, subdivisions, etc. 
2. Definition of the pairing $[\cdot, \cdot]_{\Delta}: \Gamma(\mathcal{L}) \times \Gamma_{\Delta^{0}} \mathcal{L} \rightarrow A(2 n)$. By copying the proof of Theorem 3.11 and using the previous remarks we obtain the following proposition.

Proposition 5.1. The functor $C^{\bullet}(\cdot)$ induces a natural isomorphism of A-modules

$$
\Gamma(\Delta, D \mathcal{L})=\operatorname{Hom}_{A}\left(\Gamma_{\Delta^{0}} \mathcal{L}, A(2 n)\right) .
$$

This isomorphism is independent of the choice of the volume form $\Omega$ up to multiplication by a positive real number.

Combining this isomorphism with the canonical isomorphism $\epsilon_{\Delta}$ : $\mathcal{L}_{\Delta} \rightarrow D \mathcal{L}_{\Delta}$ we obtain the following analogue of Corollary 3.16.

Proposition 5.2. There exists a natural isomorphism of A-modules

$$
\Gamma(\mathcal{L}) \rightarrow \operatorname{Hom}_{A}\left(\Gamma_{\Delta^{0}}, A(2 n)\right),
$$

hence nondegenerate pairings

$$
[\cdot, \cdot]=[\cdot, \cdot]_{\Delta}: \Gamma(\mathcal{L}) \times \Gamma_{\Delta^{0}} \mathcal{L} \rightarrow A(2 n)
$$

and

$$
(\cdot, \cdot)=(\cdot, \cdot)_{\Delta}: I H(\Delta) \times I H(\Delta, \partial \Delta) \rightarrow \mathbb{R}(2 n) .
$$

These pairings are $\mathcal{A}(\Delta)$-bilinear and are independent of the choice of the volume form up to multiplication by a positive real number.

Corollary 5.3. The graded vector spaces $I H(\Delta), I H(\Delta, \partial \Delta)$ are concentrated in degrees from 0 to $2 n$. Also $\operatorname{IH}^{2 n}(\Delta, \partial \Delta) \simeq \mathbb{R}(2 n)$.

Proof. Since the sheaf $\mathcal{L}$ is zero in negative degrees and $(\mathcal{L})^{0}=\mathbb{R}_{\Delta}$, both assertions follows from the nondegeneracy of the pairing $(\cdot, \cdot)$.

Remark 5.4. In case the fan $\Delta$ is complete, i.e. $\Delta^{0}=\Delta$, we have $\Gamma_{\Delta^{0}}(\mathcal{L})=\Gamma(\Delta, \mathcal{L})$ and the pairing of the last proposition coincides with the one in Corollary 3.16.

\section{All pairings COINCIDE in the Simplicial CASE}

Let $\Delta$ be a simplicial quasi-convex fan in $V$. Since $\mathcal{L}_{\Delta}=\mathcal{A}_{\Delta}$ we may define a pairing on $\Delta$ using Brion's functional $\zeta$. Namely define

$$
[\cdot, \cdot]_{B}: \Gamma\left(\Delta, \mathcal{A}_{\Delta}\right) \times \Gamma_{\Delta^{0}} \mathcal{A}_{\Delta} \rightarrow A(2 n), \quad[a, b]_{B}:=\zeta(a b) .
$$

Proposition 6.1 (BBFK). The pairing $[\cdot, \cdot]_{B}$ is well defined and is perfect.

This also follows from the next proposition. 
Proposition 6.2. We have $[a, b]=n ![a, b]_{B}$ for $a \in \Gamma\left(\Delta, \mathcal{A}_{\Delta}\right), b \in$ $\Gamma_{\Delta^{0}} \mathcal{A}_{\Delta}$.

Proof. Since the pairing $[\cdot, \cdot]$ is $\mathcal{A}(\Delta)$-bilinear, $[a, b]=[1, a b]$. Define the linear functional

$$
s: \Gamma_{\Delta^{0}} \mathcal{A}_{\Delta} \rightarrow A(2 n), \quad s(a):=[1, a] .
$$

It suffices to prove that $s=n ! \zeta$. We will describe the map $s$ explicitly and then compare it with $\zeta$.

Recall the Koszul resolution of the structure sheaf $\mathcal{A}_{\Delta}$. Namely, define the sheaf of vector spaces $\Omega^{1}=\Omega_{\Delta}^{1}$ as follows:

$$
\Omega_{\sigma}^{1}=\sigma^{\perp} \subset V^{*} \text {. }
$$

Put $\Omega^{i}:=\wedge^{i} \Omega^{1}$. Thus the sheaf $\Omega^{i}$ is supported on the subfan $\Delta^{\leq n-i}$; it is a sheaf of graded vector spaces which are concentrated in degree $2 i$.

We have the canonical Koszul resolution of the structure sheaf $\mathcal{A}_{\Delta}$ :

$$
0 \rightarrow A_{\Delta} \otimes \Omega^{n} \rightarrow \ldots \rightarrow A_{\Delta} \otimes \Omega^{1} \rightarrow A_{\Delta} \rightarrow \mathcal{A}_{\Delta} \rightarrow 0 .
$$

Note that $A_{\Delta} \otimes \Omega^{n}=\mathrm{D}_{\Delta}$.

From the definition of the canonical morphism $\epsilon_{\Delta}: \mathcal{A}_{\Delta} \rightarrow D \mathcal{A}_{\Delta}$ (Lemma 3.15) it is clear that $\epsilon_{\Delta}$ is the projection of the complex

$$
(*) \quad 0 \rightarrow A_{\Delta} \otimes \Omega^{n} \rightarrow \ldots \rightarrow A_{\Delta} \otimes \Omega^{1} \rightarrow A_{\Delta} \rightarrow 0
$$

on its leftmost nonzero term. Thus the map $s: \Gamma_{\Delta^{0}} \mathcal{A}_{\Delta} \rightarrow A(2 n)$ coincides with the isomorphism $\Gamma_{\Delta^{0}} \mathcal{A} \rightarrow H^{0}\left(C^{\bullet}\left(A_{\Delta} \otimes \Omega^{\bullet}\right)\right.$ ) (given by the embedding $\left.\Gamma_{\Delta^{0}} \mathcal{A} \hookrightarrow C^{\bullet}\left(A_{\Delta} \otimes \Omega^{\bullet}\right)\right)$ followed by the projection $H^{0}\left(C^{\bullet}\left(A_{\Delta} \otimes \Omega^{\bullet}\right)\right) \rightarrow C^{\bullet}\left(D_{\Delta}\right)=A(2 n)$.

First, recall the formula for the Koszul differential

$$
\begin{gathered}
\nu: A_{\Delta} \otimes \Omega^{j} \rightarrow A_{\Delta} \otimes \Omega^{j-1}: \\
\nu\left(g \otimes d x_{1} \wedge \ldots \wedge d x_{j}\right)=\sum_{i}(-1)^{i} g x_{i} \otimes d x_{1} \wedge \ldots \wedge \hat{d x_{i}} \wedge \ldots \wedge d x_{j} .
\end{gathered}
$$

The double complex $C^{\bullet}\left(A_{\Delta} \otimes \Omega^{\bullet}\right)$ looks like

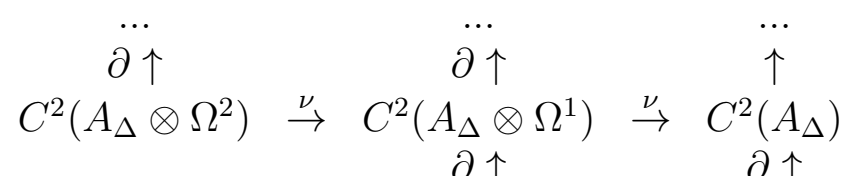

$$
\begin{aligned}
& C^{1}\left(A_{\Delta} \otimes \Omega^{1}\right) \stackrel{\nu}{\rightarrow} C^{1}\left(A_{\Delta}\right) \\
& \partial \uparrow \\
& C^{0}\left(A_{\Delta}\right)
\end{aligned}
$$


Here $\nu$ is the Koszul differential and $\partial$ is the cellular complex differential. The rows are exact except at the rightmost column. Choose $g \in \Gamma_{\Delta^{0}} \mathcal{A}_{\Delta}$. Then $g$ defines a chain $\left\{g_{\sigma}\right\} \in C^{0}\left(\mathcal{A}_{\Delta}\right)=C^{0}\left(A_{\Delta}\right)$. Put

$\tilde{s}(g):=(-1)^{[n+1 / 2]} \nu^{-1} \cdot \partial \cdot \nu^{-1} \cdot \partial \cdot \ldots \cdot \nu^{-1} \cdot \partial\left(\left\{g_{\sigma}\right\}\right) \in C^{d}\left(A_{\Delta} \otimes \Omega^{n}\right)=A \cdot \Omega$.

(The first, third, fifth, etc. composition $\nu^{-1} \cdot \partial$ is taken with the negative sign). It is clear that

$$
\tilde{s}(g)=s(g) \Omega \text {. }
$$

Let us understand the map $\tilde{s}$. Let $\xi \subset \tau$ be cones in $\Delta$ of codimension $k$ and $k+1$ respectively. Define

$$
\epsilon(\tau, \xi)= \begin{cases}1, & \text { if orientations of } \tau \text { and } \xi \text { agree; } \\ -1, & \text { otherwise. }\end{cases}
$$

Then the map on stalks $\nu^{-1} \cdot \partial:\left(A_{\Delta} \otimes \Omega^{k}\right)_{\tau} \rightarrow\left(A_{\Delta} \otimes \Omega^{k+1}\right)_{\xi}$ is equal to the wedging $\epsilon(\tau, \xi) \frac{d y}{y} \wedge$. followed by restriction from $\tau$ to $\xi$, where $y$ is any nonzero linear function on $\tau$, s.t. $\left.y\right|_{\xi}=0$.

Choose a cone $\sigma \in \Delta$ of dimension $n$. Choose linear functions $x_{1}, x_{2}, \ldots, x_{n} \in A_{\sigma}$ such that

1) $x_{i}>0$ in the interior of $\sigma$;

2) $\prod x_{i}=0$ on the boundary $\partial \sigma$;

3) $d x_{1} \wedge \ldots \wedge d x_{n}=\Omega$. (This is possible assuming $n>1$. We omit here case $n=1$, since it can be done directly.)

Let $f \in \Gamma_{\Delta^{0}} \mathcal{A}_{\Delta}$ be defined as follows

$$
f_{\tau}= \begin{cases}\prod x_{i}, & \text { if } \tau=\sigma \\ 0, & \text { otherwise }\end{cases}
$$

In particular, $f=\phi_{\sigma}$ in the notation of section 4 above.

The following lemma implies the proposition.

Lemma 6.3. We have $\tilde{s}(f)=n ! \Omega$, or, equivalently, $s(f)=n$ !.

Proof of lemma. The element $\tilde{s}(f) \in C^{n}\left(A_{\Delta} \otimes \Omega^{n}\right)$ is a sum of $n$ ! terms corresponding to the $n$ ! choices of a complete flag of faces of $\sigma$.

Claim 1. The $n$ ! summands in $\tilde{s}(f)$ are equal.

Claim 2. Each summand is equal to $\Omega$.

Clearly the claims imply the lemma. It is also clear that the only issue in proving the claims is the sign. Indeed, by applying $n$ times the formula above for $\nu^{-1} \cdot \partial$ we find that each of the $n$ ! terms is $\pm \Omega$.

Let us start with the flag $\sigma_{n}=\underline{o} \subset \sigma_{n-1} \subset \ldots \subset \sigma_{0}=\sigma$, where $\sigma_{k}=$ $\left\{x_{n-k+1}=\ldots=x_{n}=0\right\} \cap \sigma$. Assume that $\sigma_{k}$ is oriented by the form $\omega_{k}=d x_{1} \wedge \ldots \wedge d x_{n-k},\left(\omega_{n}=1\right)$. Then by definition $\epsilon\left(\sigma_{k}, \sigma_{k+1}\right) \omega_{k+1}=$ $-d x_{n-k} \wedge \omega_{k+1}$. Indeed, $-d x_{n-k}$ is the outward normal covector of $\sigma_{k+1}$ 
in $\sigma_{k}$. The sign $\epsilon\left(\sigma_{k}, \sigma_{k+1}\right)$ is negative for $k+1=n, n-2, \ldots$ Thus $\epsilon=-1$ for $[n+1 / 2]$ pairs $\sigma_{k+1} \subset \sigma_{k}$. Hence the summand of $\tilde{s}(f)$ corresponding to the above flag (with above orientaions) is equal to $(-1)^{2[n+1 / 2]} \Omega=\Omega$.

It remains to proof that the summand is independent of the flag and of the orientations of the cones of dimension $0<k<n$ in the flag.

First note that if the flag is the same, but the orientation of one of the cones, say $\sigma_{k}$, is changed $(0<k<n)$, then $\epsilon\left(\sigma_{k-1}, \sigma_{k}\right)$ and $\epsilon\left(\sigma_{k}, \sigma_{k+1}\right)$ change sign, so the corresponding summand of $\tilde{s}(f)$ remains unchanged.

Now assume that the flag is changed in one place: for some $0<k<n$ the cone $\sigma_{k}$ is replaced with $\tau_{k}:=\left\{x_{n-k}=x_{n-k+2}=\ldots=x_{n}=0\right\}$. Let $\tau_{k}$ be oriented by the form $\omega_{k}^{\prime}=d x_{1} \wedge \ldots \wedge d x_{n-k-1} \wedge d x_{n-k+1}$. (Orientations of other cones remain the same). Then

$$
\begin{gathered}
-d x_{n-k+1} \wedge \omega_{k+1}=(-1)^{n-k} \omega_{k}^{\prime}, \\
-d x_{n-k} \wedge \omega_{k}^{\prime}=(-1)^{n-k} \omega_{k-1} .
\end{gathered}
$$

Hence $\epsilon\left(\sigma_{k-1}, \tau_{k}\right)=\epsilon\left(\tau_{k}, \sigma_{k+1}\right)$. This changes the total number of minus signs among the $\epsilon$ 's by 1 , but the composition

$$
\nu^{-1} \cdot \partial \cdot \nu^{-1} \cdot \partial:\left(A_{\Delta} \otimes \Omega^{k-1}\right)_{\sigma_{k-1}} \rightarrow\left(A_{\Delta} \otimes \Omega^{k}\right)_{\tau_{k}} \rightarrow\left(A_{\Delta} \otimes \Omega^{k+1}\right)_{\sigma_{k+1}}
$$

involves wedging with $d x_{n-k+1} \wedge d x_{n-k}$ as opposed to the previous wedging with $d x_{n-k} \wedge d x_{n-k+1}$. Thus the contribution to $\tilde{s}(f)$ does not change. This proves the claims and the lemma.

Corollary 6.4. The (HL) and (HR) Theorems holds for simplicial fans. In particular, they holds if $n \leq 2$.

Proof. Indeed, this is an immediate consequence of Theorems 4.17, 4.18 and Proposition 6.2.

\section{Compatibility of the PAiRing With NATURAL OPERATions ON FANS}

1. Compatibility of the pairing with embedding of fans. Let $\Sigma$ be a fan in $V$ and $\Delta \subset \Sigma$ a subfan. Assume that both $\Sigma$ and $\Delta$ are quasi-convex. Note that $\left.\mathcal{L}_{\Sigma}\right|_{\Delta}=\mathcal{L}_{\Delta}$, hence we get a (surjective) restriction homomorphism

$$
r=r_{\Sigma, \Delta}: \Gamma\left(\Sigma, \mathcal{L}_{\Sigma}\right) \rightarrow \Gamma\left(\Delta, \mathcal{L}_{\Delta}\right) .
$$

Also note that the closed subset $\Delta^{0} \subset \Delta$ remains closed in $\Sigma$ and $\Delta^{0} \subset \Sigma^{0}$. We obtain the (injective) map

$$
s=s_{\Delta, \Sigma}: \Gamma_{\Delta^{0}} \mathcal{L}_{\Delta}=\Gamma_{\Delta^{0}} \mathcal{L}_{\Sigma} \rightarrow \Gamma_{\Sigma^{0}} \mathcal{L}_{\Sigma}
$$


Proposition 7.1. The maps $r$ and $s$ are adjoint. That is

$$
[r(a), b]_{\Delta}=[a, s(b)]_{\Sigma}
$$

for $a \in \Gamma\left(\Sigma, \mathcal{L}_{\Sigma}\right), b \in \Gamma_{\Delta^{0}} \mathcal{L}_{\Delta}$.

Proof. Note that the restriction $\left.\right|_{\Delta}$ of sheaves from $\Sigma$ to $\Delta$ commutes with the duality. Moreover, the following diagram commutes

$$
\begin{array}{ccc}
\mathcal{L}_{\Sigma} & \stackrel{\epsilon_{\Sigma}}{\rightarrow} & D\left(\mathcal{L}_{\Sigma}\right) \\
\left.\right|_{\Delta} \downarrow & & \left.\downarrow\right|_{\Delta} \\
\mathcal{L}_{\Delta} & \stackrel{\epsilon_{\Delta}}{\rightarrow} & D\left(\mathcal{L}_{\Delta}\right) .
\end{array}
$$

Also note that the restriction $\Gamma\left(\Sigma, D \mathcal{L}_{\Sigma}\right) \rightarrow \Gamma\left(\Delta, D \mathcal{L}_{\Delta}\right)$ is induced by the embedding of cellular complexes $C^{\bullet}\left(\mathcal{L}_{\Delta}\right) \hookrightarrow C^{\bullet}\left(\mathcal{L}_{\Sigma}\right)$, which in turn induces the inclusion $s: \Gamma_{\Delta^{0}} \mathcal{L}_{\Delta} \rightarrow \Gamma_{\Sigma^{0}} \mathcal{L}_{\Sigma}$. That is the following diagram commutes

$$
\begin{array}{ccc}
\Gamma\left(\Sigma, D \mathcal{L}_{\Sigma}\right) & =\operatorname{Hom}_{A}\left(\Gamma_{\Sigma} \mathcal{L}_{\Sigma}, A(2 d)\right) \\
\left.\right|_{\Delta \downarrow} \downarrow & \downarrow s^{*} \\
\left.\Gamma\left(\Delta, D \mathcal{L}_{\Delta}\right)\right) & =\operatorname{Hom}_{A}\left(\Gamma_{\Delta} \mathcal{L}_{\Delta}, A(2 d)\right) .
\end{array}
$$

This proves the proposition.

2. Compatibility of the pairing with subdivisions of fans. Let $\pi: \Psi \rightarrow \Phi$ be a subdivision of fans. Choose an admissible morphism $\alpha: \mathcal{L}_{\Phi} \rightarrow \pi_{*} \mathcal{L}_{\Psi}(3.2)$. Since $\mathcal{L}_{\Phi}$ is a direct summand of $\pi_{*} \mathcal{L}_{\Psi}$ it follows by the rigidity of $\mathcal{L}_{\Phi}(3.14)$ that $\alpha$ is a split injection.

Theorem 7.2. Let $\Delta$ be a quasi-convex fan, $\pi: \Theta \rightarrow \Delta$ - a subdivision (hence $\Theta$ is also quasi-convex). Choose an admissible morphism $\alpha$ : $\mathcal{L}_{\Delta} \rightarrow \pi_{*} \mathcal{L}_{\Theta}$ (3.2). It induces morphisms

$$
\begin{gathered}
\alpha: \Gamma_{\Delta^{0}} \mathcal{L}_{\Delta} \rightarrow \Gamma_{\Delta^{0}}\left(\pi_{*} \mathcal{L}_{\Theta}\right)=\Gamma_{\Theta^{0}} \mathcal{L}_{\Theta}, \\
\alpha: \Gamma\left(\Delta, \mathcal{L}_{\Delta}\right) \rightarrow \Gamma\left(\Delta, \pi_{*} \mathcal{L}_{\Theta}\right)=\Gamma\left(\Theta, \mathcal{L}_{\Theta}\right) .
\end{gathered}
$$

Then for $a \in \Gamma\left(\Delta, \mathcal{L}_{\Delta}\right), b \in \Gamma_{\Delta^{0}} \mathcal{L}_{\Delta}$, :

$$
(\alpha(a), \alpha(b))_{\Theta}=(a, b)_{\Delta} .
$$

Proof. First we want to "descend" the duality from $\Theta$ to $\Delta$. It was proved in $[\mathrm{BrLu}]$ that the duality functor commutes with the direct image functor $\mathbb{R} \pi_{*}$. Here we want to describe this commutation in a way that is compatible with Proposition 3.9.

Let $F \in D_{c}^{b}\left(A_{\Theta}-\bmod \right)$ and consider the cellular complexes $C_{\Theta}^{\bullet}(F)$ and $C_{\dot{\Delta}}\left(\pi_{*} F\right)$. Recall that the $n$-dimensional cones in $\Theta$ and $\Delta$ are oriented by $\Omega_{V}$. We can orient the other cones in $\Delta$ and $\Theta$ in a compatible way. Namely, if $\operatorname{dim}(\tau)=\operatorname{dim}(\pi(\tau))$ we want the orientations 
of $\tau$ and $\pi(\tau)$ to be compatible. Otherwise choose an orientation of $\tau$ at random. Then there is a natural morphism of complexes

$$
\phi(F): C_{\Delta}^{\bullet}\left(\pi_{*}(F)\right) \rightarrow C_{\Theta}^{\bullet}(F),
$$

which is compatible with identifications

$$
H^{0}\left(C_{\Theta}^{\bullet}(F)\right)=\Gamma(\Theta, F)=\Gamma\left(\Delta, \pi_{*} F\right)=H^{0}\left(C_{\Delta}^{\bullet}\left(\pi_{*} F\right)\right) .
$$

The functorial map $\phi$ above allows us to define a morphism of functors $\gamma: \mathbb{R} \pi_{*} \cdot D \rightarrow D \cdot \mathbb{R} \pi_{*}$ in the following way. Fix $F \in D_{c}^{b}\left(A_{\Theta}-\bmod \right)$. Let $F \rightarrow J^{\bullet}$ be its (finite) injective resolution and choose an injective resolution $\omega \rightarrow I^{\bullet}$ of the $A$-module $\omega$. For a subfan $\Phi \subset \Delta$ we have

$$
D \cdot \mathbb{R} \pi_{*}(F)(\Phi)=\operatorname{Hom}_{A}\left(C_{\Delta}^{\bullet}\left(\left(\pi_{*} J^{\bullet}\right)_{\Phi}\right), I^{\bullet}\right) .
$$

On the other hand, for a subfan $\Psi \subset \Theta$

$$
D(F)(\Psi)=\operatorname{Hom}_{A}\left(C_{\Theta}^{\bullet}\left(J_{\Psi}^{\bullet}\right), I^{\bullet}\right) .
$$

Note that $D(F)$ is a complex of flabby sheaves, hence $\mathbb{R} \pi_{*} D(F)=$ $\pi_{*} D(F)$. We define

$$
\gamma(F)(\Phi): \mathbb{R} \pi_{*} \cdot D(F)(\Phi) \rightarrow D \cdot \mathbb{R} \pi_{*}(F)(\Phi)
$$

to be

$$
\phi^{*}\left(J^{\bullet}\right): \operatorname{Hom}_{A}\left(C_{\Theta}^{\bullet}\left(J_{\pi^{-1}(\Phi)}^{\bullet}\right), I^{\bullet}\right) \rightarrow \operatorname{Hom}_{A}\left(C_{\Delta}^{\bullet}\left(\left(\pi_{*} J^{\bullet}\right)_{\Phi}\right), I^{\bullet}\right) .
$$

Lemma 7.3. $\gamma$ is an isomorphism of functors.

Proof. Fix a cone $\sigma \in \Delta$. It suffices to show that for an injective $A_{\Theta}$-module $J$ the map of complexes

$$
\phi(J): C^{\bullet}\left(\left(\pi_{*} J\right)_{[\sigma]}\right) \rightarrow C^{\bullet}\left(J_{\pi^{-1}([\sigma])}\right)
$$

is a quasi-isomorphism. For simplicity of notation we may assume that $\operatorname{dim}(\sigma)=n$. Put $G=\pi_{*} J$. Consider the exact sequence of sheaves

$$
0 \rightarrow G_{\partial \sigma} \rightarrow G_{[\sigma]} \rightarrow G_{\sigma} \rightarrow 0
$$

Applying Proposition 3.6 of [BrLu] to sheaves $G_{\sigma}$ and $G_{\partial \sigma}$ on fans $[\sigma]$ and $\partial \sigma$ respectively we obtain natural quasi-isomorphisms

$$
\mathbb{R} \Gamma\left([\sigma], G_{\sigma}\right)=C_{\Delta}^{\bullet}\left(G_{\sigma}\right), \quad \mathbb{R} \Gamma\left(\partial \sigma, G_{\partial \sigma}\right)=C_{\Delta}^{\bullet}\left(G_{\partial \sigma}\right)[1] .
$$

The sheaf $G$ is injective, hence the exact triangle

$$
C_{\Delta}^{\bullet}\left(G_{[\sigma]}\right) \rightarrow C_{\Delta}^{\bullet}\left(G_{\sigma}\right) \rightarrow C_{\Delta}^{\bullet}\left(G_{\partial \sigma}\right)[1]
$$

is quasi-isomorphic to the (middle part of the) short exact sequence

$$
0 \rightarrow \Gamma_{\sigma} G \rightarrow \Gamma([\sigma], G) \rightarrow \Gamma(\partial \sigma, G) \rightarrow 0 .
$$


The same arguments show that the triangle

$$
C_{\Theta}^{\bullet}\left(J_{\pi^{-1}([\sigma])}\right) \rightarrow C_{\Theta}^{\bullet}\left(J_{\pi^{-1}(\sigma)}\right) \rightarrow C_{\Theta}^{\bullet}\left(J_{\pi^{-1}(\partial \sigma)}\right)[1]
$$

is quasi-isomorphic to the (middle part of the) short exact sequence

$$
0 \rightarrow \Gamma_{\pi^{-1}(\sigma)} J \rightarrow \Gamma\left(\pi^{-1}([\sigma]), J\right) \rightarrow \Gamma\left(\pi^{-1}(\partial \sigma), J\right) \rightarrow 0 .
$$

The short exact sequences (1) and (2) are isomorphic and the isomorphism $\Gamma_{\sigma} G \simeq \Gamma_{\pi^{-1}(\sigma)} J$ coincides with the map $\phi(J)$ under the above quasi-isomorphisms.

Remark 7.4. As in the proof of Lemma 3.15 it is easy to see that the stalks $\left(\pi_{*} D \mathcal{L}_{\Theta}\right)_{\underline{o}}$ and $\left(D \pi_{*} \mathcal{L}_{\Theta}\right)_{\underline{o}}$ are canonically isomorphic to $\mathbb{R}$. Under this identifications $\gamma\left(\mathcal{L}_{\Theta}\right)_{\underline{o}}=i \bar{d}$.

Consider the diagram of sheaves

$$
\begin{aligned}
& \pi_{*} \mathcal{L}_{\Theta} \stackrel{\pi_{*} \epsilon \Theta}{\rightarrow} \pi_{*} D \mathcal{L}_{\Theta} \stackrel{\gamma}{\rightarrow} D \pi_{*} \mathcal{L}_{\Theta} \\
& \alpha \uparrow \quad \downarrow D(\alpha) \\
& \mathcal{L}_{\Delta} \quad \stackrel{\epsilon_{\Delta}}{\rightarrow} \quad D \mathcal{L}_{\Delta} .
\end{aligned}
$$

Lemma 7.5. The above diagram commutes.

Proof. Note that the stalks at $\underline{o}$ of all the sheaves are canonically isomorphic to $\mathbb{R}$ and all the morphisms are equal to $i d$ at $\underline{o}$. Hence the diagram commutes by the rigidity of $\mathcal{L}_{\Delta}$.

It follows from the last two lemmas that the following diagram commutes

$$
\begin{array}{ccccc}
\Gamma\left(\Delta, \pi_{*} \mathcal{L}_{\Theta}\right) \stackrel{\pi_{*} \epsilon_{\Theta}}{\rightarrow} & \Gamma\left(\Delta, \pi_{*} D \mathcal{L}_{\Theta}\right) & = & \mathbb{R} \operatorname{Hom}_{A}\left(C_{\Theta}^{\bullet}\left(\mathcal{L}_{\Theta}\right), A(2 d)\right) \\
& & \gamma \downarrow & & \phi\left(\mathcal{L}_{\Theta}\right)^{*} \\
\alpha \uparrow & & \Gamma\left(\Delta, D \pi_{*} \mathcal{L}_{\Theta}\right) & = & \mathbb{R} \operatorname{Hom}_{A}\left(C_{\Delta}^{\bullet}\left(\pi_{*} \mathcal{L}_{\Theta}\right), A(2 d)\right) \\
& & D(\alpha) \downarrow & & \downarrow C_{\Delta}^{\bullet}(\alpha)^{*} \\
\Gamma\left(\Delta, \mathcal{L}_{\Delta}\right) & \stackrel{\epsilon_{\Delta}}{\rightarrow} & \Gamma\left(\Delta, D \mathcal{L}_{\Delta}\right) & = & \mathbb{R} \operatorname{Hom}_{A}\left(C_{\Delta}^{\bullet}\left(\mathcal{L}_{\Delta}\right), A(2 d)\right) .
\end{array}
$$

Finally note the commutativity of the natural diagram

$$
\begin{array}{ccc}
\Gamma_{\Delta^{0}} \mathcal{L}_{\Delta} & \stackrel{\alpha}{\rightarrow} & \Gamma_{\Theta^{0}} \mathcal{L}_{\Theta} \\
\downarrow & & \downarrow \\
C_{\Delta}^{\bullet}\left(\mathcal{L}_{\Delta}\right) & \stackrel{\phi\left(\mathcal{L}_{\Theta}\right) \cdot C_{\Delta}^{\bullet}(\alpha)}{\rightarrow} & C_{\Theta}^{\bullet}\left(\mathcal{L}_{\Theta}\right) .
\end{array}
$$

The theorem follows.

Corollary 7.6. For a complete fan $\Phi$ the pairing

$$
[\cdot, \cdot]: \mathcal{A}(\Phi) \times \mathcal{A}(\Phi) \rightarrow A(2 d)
$$

is symmetric. 
Proof. This is true if the fan $\Phi$ is simplicial (Proposition 6.2). For a general fan $\Phi$ take a subdivision $\pi: \Psi \rightarrow \Phi$ where $\Psi$ is simplicial and use the last theorem.

Corollary 7.7. Let $\Phi$ be a complete fan. Let $a, b \in \Gamma\left(\mathcal{L}_{\Phi}\right)$ have disjoint supports. Then $[a, b]_{\Phi}=0$.

Proof. Same as that of the last corollary.

3. Local-global compatibility of the pairing. Assume that $\Delta=$ $\operatorname{St}(\rho)$ for a 1-dimensional cone $\rho \in \Delta^{0}$. Denote by $p: V \rightarrow \bar{V}:=V /\langle\rho\rangle$ the projection along $\rho$. Then $\Phi:=p(\partial \Delta)$ is a complete fan in $\bar{V}$. We want to relate the pairings on $\Delta$ and on $\Phi$.

Denote $B=\operatorname{Sym} \bar{V}^{*}$. By Lemma 3.18 there is a canonical isomorphism of $A$-modules $A \otimes_{B} \Gamma\left(\Phi_{\rho}, \mathcal{L}_{\Phi}\right) \simeq \Gamma\left(\Delta, \mathcal{L}_{\Delta}\right)$, hence an identification $I H(\Phi)=I H(\Delta)$.

Let $x_{n}$ be a linear function on $V$ which is positive on the interior $\rho^{0}$ of $\rho$. We have $A=B\left[x_{n}\right]$. The pairing on $I H(\Phi)$ is defined once we choose a volume form $\Omega_{\bar{V}}$ on $\bar{V}$. Choose it to satisfy

$$
\Omega_{V}=d x_{n} \wedge \Omega_{\bar{V}}
$$

Let $\psi=x_{n}-f=0$ be the equation of the boundary $\partial \Delta$, where $f$ is a piecewise linear function on $\Phi$. Multiplication by $\psi$ maps $\Gamma\left(\Delta, \mathcal{L}_{\Delta}\right)$ to $\Gamma_{\Delta^{0}} \mathcal{L}_{\Delta}$, hence it maps $I H(\Delta)$ to $I H(\Delta, \partial \Delta)$.

Proposition 7.8. Let $a, b \in I H(\Phi)=I H(\Delta)$. Then

$$
n(a, b)_{\Phi}=(a, \psi(b))_{\Delta} .
$$

Proof. Step 1: Reduction to the simplicial case. Let $\bar{\pi}: \Psi \rightarrow \Phi$ be a subdivision, such that the fan $\Psi$ is simplicial. It induces the subdivision $\pi: \Theta \rightarrow \Delta$, such that $\Theta=\operatorname{St}(\rho)$ and $p(\partial \Theta)=\Psi$. Then $\Theta$ is also simplicial. Choose an admissible embedding $\bar{\alpha}: \mathcal{L}_{\Phi} \rightarrow \bar{\pi}_{*} \mathcal{L}_{\Psi}$. It induces an admissible embedding $\alpha: \mathcal{L}_{\Delta} \rightarrow \pi_{*} \mathcal{L}_{\Theta}$. By Theorem 7.2 the induces maps on global sections are isometries (and $\alpha$ commutes with multiplication by $\psi$ ). Thus it suffices to prove the proposition for $\Theta$, i.e. we may assume that $\Delta$ is simplicial.

Step 2. By Proposition 6.2 above for $y \in \mathcal{A}(\Delta), z \in \Gamma_{\Delta^{0}} \mathcal{A}_{\Delta}$

$$
[y, z]_{\Delta}=n ! \zeta(y z)
$$

where $\zeta: \Gamma_{\Delta^{0}} \mathcal{A}_{\Delta} \rightarrow A(2 n)$ is the Brion functional determined by the volume form $\Omega_{V}$. Similarly, for $s, t \in \mathcal{A}(\Phi)$

$$
[s, t]_{\Phi}=(n-1) ! \bar{\zeta}(s t)
$$


for the functional $\bar{\zeta}: \mathcal{A}(\Phi) \rightarrow B(2(n-1))$, determined by $\Omega_{\bar{V}}$. Thus we must check that for a function $g \in \mathcal{A}(\Phi)$ we have

$$
\bar{\zeta}(g)=\zeta(\psi g) \text {. }
$$

It suffices to check the last equality for $g=\phi_{\sigma}$ (section 4), where $\sigma \in \Phi$ is of dimension $n-1$. Then $\psi g=\phi_{\tau}$, where $\tau \in \operatorname{Star}^{0}(\rho)$ is the preimage of $\sigma$ (by our choice of the volume forms). So $\bar{\zeta}(g)=1=\zeta(\psi g)$ by Theorem 4.12 .

Corollary 7.9. Multiplication by $\psi$ induces isomorphisms $\Gamma\left(\mathcal{L}_{\Delta}\right) \rightarrow$ $\Gamma_{\Delta^{0}} \mathcal{L}_{\Delta}, I H(\Delta) \rightarrow I H(\Delta, \partial \Delta)$.

Proof. This follows from the last proposition and the nondegeneracy of the pairings $(\cdot, \cdot)_{\Delta}$ and $(\cdot, \cdot)_{\Phi}$. Actually, one can also see this directly by using a pl-isomorphism of fans $\Delta$ and $\Phi \times \rho$, defined by the function $f$.

\section{Some immEDiATE APPLiCATIONS AND GENERALIZATIONS}

1. Let $\Phi$ be a complete fan in $V, \Delta \subset \Phi$ - a subfan which is quasiconvex. Then the fan $\Sigma:=\Phi-\Delta^{0}$ is also quasi-convex, since $\partial \Delta=\partial \Sigma$. Consider the exact sequences of free $A$-modules

$$
\begin{gathered}
0 \rightarrow \Gamma_{\Delta^{0}} \mathcal{L}_{\Phi} \rightarrow \Gamma\left(\Phi, \mathcal{L}_{\Phi}\right) \rightarrow \Gamma\left(\Sigma, \mathcal{L}_{\Phi}\right) \rightarrow 0 \\
0 \rightarrow \Gamma_{\Sigma^{0}} \mathcal{L}_{\Phi} \rightarrow \Gamma\left(\Phi, \mathcal{L}_{\Phi}\right) \rightarrow \Gamma\left(\Delta, \mathcal{L}_{\Phi}\right) \rightarrow 0 .
\end{gathered}
$$

The pairings $[\cdot, \cdot]$ on the three fans identify the terms of the second sequence as the duals (in the sense of $\operatorname{Hom}_{A}(\cdot, A(2 n))$ of the corresponding terms of the first one. It follows from Proposition 7.1 that the whole second sequence is the dual of the first one, i.e. the maps in the second sequence are adjoint to the maps in the first.

2. Let us translate Timorin's Theorem 4.7 to the fans setting. Given a complete simplicial fan $\Phi$ choose a convex simple $n$-dimensional polytope $P \subset W$ so that $\Phi=\Phi_{P}$. Let $P_{i} \subset P$ be one of the $n-1$ dimensional faces and $\rho_{i} \in \Phi$ - the corresponding 1-dimensional cone. Then the differential operator $\partial_{i} \in A_{1}(P)$ corresponds to a piecewise linear function $\lambda_{i}$ on $\Phi$ that takes nonzero values on $\rho_{i}$ and is zero on every other 1-dimensional cone. Given a face of $F=P_{i_{1}} \cap \ldots \cap P_{i_{k}}$ of $P$ the product $\lambda_{i_{1}} \cdot \ldots \cdot \lambda_{i_{k}}$ is supported on the $\operatorname{star} \operatorname{St}\left(\sigma_{F}\right)$. As an immediate corollary of Theorem 4.7 and Proposition 7.1 we obtain the following proposition. 
Proposition 8.1. For a complete simplicial fan $\Phi$ the space $H^{2 k}(\Phi)=$ $I H^{2 k}(\Phi)$ has a basis consisting of (residues of) functions $f \in \mathcal{A}(\Phi)$ such that the support of $f$ is contained in $\operatorname{St}(\sigma)$ for $\sigma \in \Phi$ of dimension $k$. In particular, the map

$$
\bigoplus_{\operatorname{dim}(\sigma)=1} H^{\bullet}([\operatorname{St}(\sigma)], \partial \operatorname{St}(\sigma)) \rightarrow \bigoplus_{k>0} H^{2 k}(\Phi)
$$

is surjective. Hence the dual map

$$
\bigoplus_{k<n} H^{2 k}(\Phi) \rightarrow \bigoplus_{\operatorname{dim}(\sigma)=1} H^{\bullet}([\operatorname{St}(\sigma)])
$$

is injective.

3. Following $[\mathrm{Ka}]$ we present a simple argument which deduces the (HL) theorem for simplicial fans of dimension $n$ from the (HR) theorem for simplicial fans of dimension $n-1$.

Let $\Phi$ be a complete simplicial fan in $V$ and $l$ be a strictly convex piecewise linear function on $\Phi$. After adding a globally linear function to it we may assume that $l$ is strictly positive on $V-0$. For each 1dimensional cone $\rho_{i} \in \Phi$ consider the $(n-1)$-dimensional complete fan $\Phi_{i}=p_{i}\left(\partial \operatorname{St}\left(\rho_{i}\right)\right)$ in $V_{i}=V /\left\langle\rho_{i}\right\rangle$ (where $p_{i}: V \rightarrow V_{i}$ is the projection). Since the fan $\Phi$ is simplicial we can write (uniquely)

$$
l=\sum_{i} \lambda_{i}
$$

where $\lambda_{i}$ is a piecewise linear function on $\Phi$ supported on $\operatorname{St}\left(\rho_{i}\right)$.

Suppose $h \in I H^{n-k}(\Phi)$ is such that

$$
l^{k} \cdot h=0 .
$$

Then

$$
0=\left(h, l^{k} h\right)_{\Phi}=\sum_{i}\left(h, \lambda_{i} l^{k-1} h\right)_{\Phi} .
$$

Fix a 1-dimensional cone $\rho_{i} \in \Phi$. Let $h_{i} \in I H\left(\left[\operatorname{St}\left(\rho_{i}\right)\right]\right)$ denote the restriction of $h$ to $\operatorname{St}\left(\rho_{i}\right)$. Changing $l$ by a global linear function (which depends on $i$ ) we may assume that $\left.l\right|_{\operatorname{St}\left(\rho_{i}\right)}=p_{i}^{*}\left(l_{i}\right)$ for a strictly convex function $l_{i}$ on $\Phi_{i}$. Identifying $I H\left(\left[\operatorname{St}\left(\rho_{i}\right)\right]\right)=I H\left(\Phi_{i}\right)$ as in Lemma 3.18 we find that

$$
h_{i} \in \operatorname{Prim}_{l_{i}} I H\left(\Phi_{i}\right) .
$$

Since $\lambda_{i}$ is supported on $\operatorname{St}\left(\rho_{i}\right)$ by Proposition 7.1

$$
\left(h, \lambda_{i} l^{k-1} h\right)_{\Phi}=\left(h_{i}, \lambda_{i} l^{k-1} h_{i}\right)_{\left[\operatorname{St}\left(\rho_{i}\right)\right]} .
$$

Now apply Proposition 7.8 to get

$$
\left(h_{i}, \lambda_{i} l^{k-1} h_{i}\right)_{\left[\operatorname{St}\left(\rho_{i}\right)\right]}=n\left(h_{i}, l_{i}^{k-1} h_{i}\right)_{\Phi_{i}} .
$$


By the (HR) theorem for $\Phi_{i},(-1)^{\frac{n-k}{2}}\left(h_{i}, l^{k-1} h_{i}\right)_{\Phi_{i}}>0$. It follows that $h_{i}=0$ for all $i$. But then by Proposition $8.1 h=0$.

This argument can be used to prove the following corollary

Corollary 8.2 (Ka). Let $\Phi$ be a complete $n$-dimensional fan with a strictly convex function l. Assume that $\Phi^{s} \subset[\sigma]$ for a unique cone $\sigma \in \Phi$. Let $h \in I H^{n-k}(\Phi)$ be such that $l^{k} h=0$. Then the (HR) theorem in dimension $n-1$ implies that the restriction $h_{i} \in I H^{n-k}\left(\left[\operatorname{St}\left(\rho_{i}\right)\right]\right)$ is zero for all 1-dimensional cones $\rho_{i} \in \Phi-[\sigma]$.

Proof. Let $\rho_{i}$ be a 1-dimensional cone in $\Phi-[\sigma]$. Then as in the previous argument (and using the same notation) we find that

$$
h_{i} \in \operatorname{Prim}_{l_{i}} I H^{n-k}\left(\Phi_{i}\right) .
$$

By adding a global linear function to $l$ we may assume that $\left.l\right|_{\sigma}=0$ and $l(x)>0$ for $x \notin \sigma$. Then as before we can write

$$
l=\sum_{i} \lambda_{i}
$$

where the summation is over all $\rho_{i} \in \Phi-[\sigma]$. Then again

$$
0=\left(h, l^{k} h\right)_{\Phi}=\sum_{i}\left(h, \lambda_{i} l^{k-1} h\right)_{\Phi}=n \sum_{i}\left(h_{i}, l_{i}^{k-1} h_{i}\right)_{\Phi_{i}} .
$$

If $h_{i} \neq 0$, then by (HR) theorem for $\Phi_{i},(-1)^{\frac{n-k}{2}}\left(h_{i}, l_{i}^{k-1} h_{i}\right)_{\Phi_{i}}>0$. This proves the corollary.

4. Sometimes it is useful to have a pairing on $\Gamma\left(\mathcal{L}_{\Delta}\right)$ for a quasiconvex fan. The next proposition follows easily from the results about the usual pairing. We denote by $Q(A)$ the localization of $A$ with respect to all nonzero homogeneous polynomials.

Proposition 8.3. Let $\Delta$ be a quasi-convex fan in $V$. There exists a unique $\mathcal{A}(\Delta)$-bilinear pairing

$$
\{\cdot, \cdot\}_{\Delta}: \Gamma\left(\mathcal{L}_{\Delta}\right) \times \Gamma\left(\mathcal{L}_{\Delta}\right) \rightarrow Q(A)(2 n),
$$

which has the following properties.

a) The restriction of $\{\cdot, \cdot\}_{\Delta}$ to $\Gamma\left(\mathcal{L}_{\Delta}\right) \times \Gamma_{\Delta^{0}} \mathcal{L}_{\Delta}$ takes values in $A(2 n)$ and coincides with $[\cdot, \cdot]_{\Delta}$. In particular, if $\Delta$ is complete then $\{\cdot, \cdot\}_{\Delta}=$ $[\cdot, \cdot]_{\Delta}$.

b) Assume that the fan $\Delta$ is covered by quasi-convex subfans $\Delta_{i}$ so that every cone in $\Delta$ of maximal dimension belongs to a unique $\Delta_{i}$. Then the restriction map

$$
\Gamma\left(\mathcal{L}_{\Delta}\right) \rightarrow \bigoplus_{i} \Gamma\left(\mathcal{L}_{\Delta_{i}}\right)
$$


is an isometry. That is, if $a_{i} \in \Gamma\left(\mathcal{L}_{\Delta_{i}}\right)$ denotes the image of $a \in \Gamma\left(\mathcal{L}_{\Delta}\right)$ then

$$
\{a, b\}_{\Delta}=\sum_{i}\left\{a_{i}, b_{i}\right\}_{\Delta_{i}}
$$

c) Let $\pi: \Sigma \rightarrow \Delta$ be a subdivision. Then an admissible morphism $\alpha: \mathcal{L}_{\Delta} \rightarrow \pi_{*} \mathcal{L}_{\Sigma}$ defines an isometry $\alpha: \Gamma\left(\mathcal{L}_{\Delta}\right) \rightarrow \Gamma\left(\pi_{*} \mathcal{L}_{\Sigma}\right)$ with respect to the pairings $\{\cdot, \cdot\}$.

d) Let $\Phi$ be a complete fan with a covering $\Phi=\cup_{i} \Delta_{i}$ by quasi-convex subfans $\Delta_{i}$, such that every cone in $\Phi$ of maximal dimension belongs to a unique $\Delta_{i}$. Then the restriction map

$$
\Gamma\left(\mathcal{L}_{\Phi}\right) \rightarrow \bigoplus_{i} \Gamma\left(\mathcal{L}_{\Delta_{i}}\right)
$$

is an isometry. That is if $a_{i} \in \Gamma\left(\mathcal{L}_{\Delta_{i}}\right)$ denotes the image of $a \in \Gamma\left(\mathcal{L}_{\Phi}\right)$, then

$$
[a, b]_{\Phi}=\sum_{i}\left\{a_{i}, b_{i}\right\}_{\Delta_{i}}
$$

Proof. Let us first prove uniqueness. Indeed, since the pairing $[\cdot, \cdot]_{\Delta}$ is nondegenerate, the free $A$-modules $\Gamma_{\Delta^{0}} \mathcal{L}_{\Delta}$ and $\Gamma\left(\mathcal{L}_{\Delta}\right)$ have the same rank. Hence the $A$-module $\Gamma\left(\mathcal{L}_{\Delta}\right) / \Gamma_{\Delta^{0}} \mathcal{L}_{\Delta}$ is torsion. Since the $A$ module $Q(A)(2 n)$ is torsion free it follows that a pairing $\{\cdot, \cdot\}_{\Delta}$ satisfying a) is unique.

The construction of the pairing $\{\cdot, \cdot\}$ is the "same" as that of $[\cdot, \cdot]$. Assume first that the fan $\Delta$ is simplicial, i.e. $\mathcal{L}_{\Delta}=\mathcal{A}_{\Delta}$. Then put

$$
\{f, g\}_{\Delta}=\frac{1}{n !} \zeta(f g) \in Q(A)
$$

where $\zeta$ is the Brion functional. As was explained above this definition is forced on us if we want the property a) to hold. For a general quasiconvex fan $\Delta$ choose a subdivision $\pi: \Sigma \rightarrow \Delta$ with a simplicial $\Sigma$ and an admissible morphism $\alpha: \mathcal{L}_{\Delta} \rightarrow \pi_{*} \mathcal{L}_{\Sigma}$. Put

$$
\{a, b\}_{\Delta}:=\{\alpha(a), \alpha(b)\}_{\Sigma} .
$$

Here again we had no choice if the property c) is to hold. Theorem 7.2 implies that the property a) holds, which in turn means that the pairing $\{\cdot, \cdot\}_{\Delta}$ is independent of the choice of the subdivision $\pi$ and the admissible morphism $\alpha$.

The properties b) and c) are easy to check and d) is a special case of b).

Example 8.4. In the above proposition one can take the "affine" fan $\Delta=[\sigma]$ for a cone $\sigma$ of dimension $n$. This gives the pairing on the 
stalk at the closed point

$$
\{\cdot, \cdot\}_{[\sigma]}: \mathcal{L}_{[\sigma], \sigma} \times \mathcal{L}_{[\sigma], \sigma} \rightarrow Q(A)(2 n) .
$$

5. Consider a complete fan $\Phi$ in $V$ with the following two properties: 1) $\Phi$ contains a 1-dimensional cone $\rho$ and its negative $\rho^{\prime}:=-\rho$, and it has a local product structure at these cones; 2) The quasi-convex subfans $\Delta^{+}=[\operatorname{St}(\rho)]$ and $\Delta^{-}=\left[\operatorname{St}\left(\rho^{\prime}\right)\right]$ intersect along the common boundary and $\Phi=\Delta^{+} \cup \Delta^{-}$.

Let $p: V \rightarrow \bar{V}=V /\langle\rho\rangle$ be the projection and $\Phi_{\rho}=p(\partial \operatorname{St}(\rho))$ the complete fan in $\bar{V}$. Put $B=\operatorname{Sym} \bar{V}^{*}$. By Lemma 3.18 the map $p$ induces natural isomorphisms

$$
\Gamma\left(\mathcal{L}_{\Delta^{-}}\right) \simeq A \otimes_{B} \Gamma\left(\mathcal{L}_{\Phi_{\rho}}\right) \simeq \Gamma\left(\mathcal{L}_{\Delta^{+}}\right) .
$$

Hence we obtain the isomorphism of $A$-modules $\gamma: \Gamma\left(\mathcal{L}_{\Delta^{-}}\right) \rightarrow \Gamma\left(\mathcal{L}_{\Delta^{+}}\right)$.

Lemma 8.5. For $a, b \in \Gamma\left(\mathcal{L}_{\Delta^{-}}\right)$we have

$$
\{a, b\}_{\Delta^{-}}=-\{\gamma(a), \gamma(b)\}_{\Delta^{+}} .
$$

Proof. Let $\pi_{\rho}: \Psi_{\rho} \rightarrow \Phi_{\rho}$ be a subdivision with a simplicial $\Psi_{\rho}$. This induces subdivisions $\pi_{-}: \tilde{\Delta}^{-} \rightarrow \Delta^{-}, \pi_{+}: \tilde{\Delta}^{+} \rightarrow \Delta^{+}$with simplicial $\tilde{\Delta}^{-}$and $\tilde{\Delta}^{+}$. An admissible morphism $\alpha_{\rho}: \mathcal{L}_{\Phi_{\rho}} \rightarrow \pi_{\rho *} \mathcal{L}_{\Psi_{\rho}}$ induces corresponding admissible morphisms $\alpha_{-}$and $\alpha_{+}$. The induces morphisms $\alpha_{-}$and $\alpha_{+}$on global sections commute with the isomorphism $\gamma$. Hence by Proposition $8.3 \mathrm{c}$ ) we may assume that the fan $\Phi$ is simplicial. But then the lemma follows from the definition of the Brion functional $\zeta$.

\section{Proof of Hodge-Riemann and Hard Lefschetz theorems}

We assume that (HL) and (HR) theorems hold for fans of dimension $\leq n-1$.

Let $\Phi$ be a complete projective fan of dimension $n$ in $V$. Consider its singular subfan $\Phi^{s}$. If $\Phi^{s}$ is empty then $\Phi$ is simplicial and the (HL) and (HR) theorems hold for $\Phi$ (Corollary 6.4). Otherwise choose a maximal cone $\sigma \in \Phi^{s}$ and a ray $\rho \in \sigma^{0}$ and consider the corresponding star subdivision

$$
\pi: \Psi \rightarrow \Phi
$$

(Example 2.5). The fan $\Psi$ is also projective (Lemma 2.13) and is "less" singular than $\Phi$, i.e. $\Psi^{s}$ contains a smaller number of cones than $\Phi^{s}$. So by induction on the size of the singular subfan we may assume that (HL) and (HR) theorems hold for $\Psi$. We are going to deduce from this that the theorems hold for $\Phi$. Choose a strictly convex piecewise linear function $l$ on $\Phi$. 
Let $\tilde{l}$ be a piecewise function on $\Psi$ with support in $\operatorname{St}_{\Psi}(\rho)$ such that $\left.\tilde{l}\right|_{\rho}<0$. By Lemma 2.13 if we choose $\tilde{l}$ sufficiently small then $\hat{l}=l+\tilde{l}$ is strictly convex on $\Psi$. Fix one such $\hat{l}$.

Let $p: V \rightarrow V /\langle\rho\rangle=\bar{V}$ be the projection. Then $p(\partial \operatorname{St}(\rho))=$ $\Phi_{\rho}$ is a complete fan in $\bar{V}$. By Lemma 3.18 the projection $p$ induces an isomorphism of $\mathcal{A}\left(\Phi_{\rho}\right)$-modules $I H\left(\Phi_{\rho}\right)=I H([\operatorname{St}(\rho)])$. Put $B=$ Sym $\bar{V}^{*}$.

Case 1: Assume that $\operatorname{dim}(\sigma)=n$. We want to do this special case first, because it follows almost immediately from the functorial properties of the pairing $(\cdot, \cdot)$, and the main ideas are transparent.

Choose an admissible embedding $\alpha: \mathcal{L}_{\Phi} \rightarrow \pi_{*} \mathcal{L}_{\Psi}$. This induces an isometry $\alpha: I H(\Phi) \hookrightarrow I H(\Psi)$. We claim that one can choose $\alpha$ so that $\alpha\left(\operatorname{Prim}_{l} I H(\Phi)\right) \subset \operatorname{Prim}_{\hat{l}} I H(\Psi)$. This is proved in Lemma 9.2 below.

Choose linear coordinates $x_{1}, \ldots, x_{n}$ on $V$ so that the first $n-1$ vanish on $\rho$ and $x_{n}$ is negative on $\operatorname{St}(\rho)$. Then $\tilde{l}=x_{n}+f\left(x_{1}, \ldots, x_{n-1}\right)$ where $f$ is a strictly convex (because the cone $\sigma$ is convex) piecewise linear function on $\Phi_{\rho}$. Therefore the $\tilde{l}$-action on $I H([\operatorname{St}(\rho)])$ coincides with the $f$-action on $I H\left(\Phi_{\rho}\right)$, so, in particular,

$$
\tilde{l}^{i}: I H^{n-1-i}([\operatorname{St}(\rho)]) \rightarrow I H^{n-1+i}([\operatorname{St}(\rho)])
$$

is an isomorphism.

Lemma 9.1. We may choose an admissible embedding $\alpha: \mathcal{L}_{\Phi} \hookrightarrow \pi_{*} \mathcal{L}_{\Psi}$ so that the image of $\overline{\mathcal{L}_{\Phi, \sigma}}$ in $I H([\mathrm{St}(\rho)])$ is equal to the $\tilde{l}$-primitive subspace.

Proof. This is clear from the definition of the sheaf $\mathcal{L}_{\Phi}$.

Lemma 9.2. Under an admissible embedding $\alpha$ as in the last lemma the image of $\operatorname{Prim}_{l} I H(\Phi)$ is contained in $\operatorname{Prim}_{\tilde{l}} I H(\Psi)$.

Proof. For simplicity of notation we identify $I H(\Phi)$ as a subspace of $I H(\Psi)$ (by means of $\alpha$ ).

Choose $a \in \operatorname{Prim}_{l} I H^{n-k}(\Phi)$. We have

$$
\hat{l}^{k+1} a=l^{k+1} a+\tilde{l} q(l, \tilde{l}) a=\tilde{l} q(l, \tilde{l}) a
$$

for a polynomial $q$. Since the support of $\tilde{l}$ is contained in $\operatorname{St}(\rho)$ and $l l_{\operatorname{St}(\rho)}$ is linear we have $\tilde{l} l a=0$. Thus

$$
\hat{l}^{k+1} a=\tilde{l}^{k+1} a
$$

may be considered as an element of $I H([\operatorname{St}(\rho)], \partial \operatorname{St}(\rho))$ (which is a subspace of $I H(\Psi))$. We have $\tilde{l}^{k+1} a=0$ if $\left(c, \widetilde{l}^{k+1} a\right)_{[\operatorname{St}(\rho)]}=0$ for all 
$c \in I H([\operatorname{St}(\rho)]$. By Proposition 7.8

$$
\left(c, \tilde{l}^{k+1} a\right)_{[\operatorname{St}(\rho)]}=-n\left(c, f^{k} a\right)_{\Phi_{\rho}}=0,
$$

because $f^{k} a=0$.

Let us fix an admissible embedding $\alpha$ as in Lemma 9.1 above and choose $0 \neq a \in \operatorname{Prim}_{l} I H^{n-k}(\Phi)$. Then by Lemma 9.2 and by our induction hypothesis

$$
(-1)^{\frac{n-k}{2}}\left(a, \hat{l}^{k} a\right)_{\Psi}>0
$$

We have

$$
\begin{aligned}
\left(a, \hat{l}^{k} a\right)_{\Psi} & =\left(a, l^{k} a\right)_{\Psi}+(a, \tilde{l} t(l, \tilde{l}) a)_{\Psi} \\
& =\left(a, l^{k} a\right)_{\Phi}+(a, \tilde{l} t(l, \tilde{l}) a)_{\Psi}
\end{aligned}
$$

for a polynomial $t$. By the support consideration the element $\tilde{l} t(l, \tilde{l}) a$ belongs to the subspace $I H([\operatorname{St}(\sigma)], \partial \operatorname{St}(\sigma)) \subset I H(\Psi)$. By abuse of notation we will denote also by $a$ the image of $a$ in $I H([\operatorname{St}(\sigma)])$. Then by Propositions 7.1 and 7.8

$$
\begin{aligned}
(a, \tilde{l} t(l, \tilde{l}) a)_{\Psi} & =(a, \tilde{l} t(l, \tilde{l}) a)_{[\operatorname{St}(\sigma)]} \\
& =\left(a, \tilde{l}^{k} a\right)_{[\operatorname{St}(\sigma)]} \\
& =-n\left(a, f^{k-1} a\right)_{\Phi_{\rho}}
\end{aligned}
$$

By Lemma 9.1 the element $a \in I H^{n-k}\left(\Phi_{\rho}\right)$ is $f$-primitive. Hence by (HR) theorem for $\Phi_{\rho}$

$$
(-1)^{\frac{n-k}{2}}\left(a, f^{k-1} a\right)_{\Phi_{\rho}} \geq 0
$$

(the inequality is not strict since $a$ may be zero in $I H^{n-k}\left(\Phi_{\rho}\right)$ ). Therefore

$$
(a, \tilde{l} t(l, \tilde{l}) a)_{\Psi} \leq 0
$$

and

$$
(-1)^{\frac{n-k}{2}}\left(a, l^{k} a\right)_{\Phi}>0
$$

which proves the (HL) and (HR) theorems for $\Phi$.

Remark 9.3. Our proof shows that the quadratic form $Q_{\hat{l}}$ on $I H(\Psi)$ tends to be more degenerate than the form $Q_{l}$ on $I H(\Phi)$. Indeed, this is what happens in blow-ups of algebraic varieties. We will also see this in the remaining case below.

Case 2: $\operatorname{dim}(\sigma)<n$. Here we essentially copy [Ka].

Denote $\Delta=\left[\operatorname{St}_{\Phi}(\sigma)\right], \rho^{\prime}=-\rho$. Consider the "complementary" fan

$$
\Delta^{\prime}:=\left\{\rho^{\prime}+\tau \mid \tau \in \partial \Delta\right\}
$$


The (quasi-convex) fans $\Delta$ and $\Delta^{\prime}$ intersect along the common boundary and their union is a complete fan in $V$ which we denote $\bar{\Delta}$. First we prove the (HR) theorem for this auxiliary fan $\bar{\Delta}$ and then use a trick to deduce the (HR) theorem for $\Phi$.

Denote $\hat{\Delta}=\left[\operatorname{St}_{\Psi}(\rho)\right]$ (so that $|\Delta|=|\hat{\Delta}|$ ).

By changing $\hat{l}$ by a global linear function we may assume that $\hat{l}_{\rho}=0$ and hence $\hat{l}_{\hat{\Delta}}=p^{*} l_{\rho}$ for a strictly convex function $l_{\rho}$ on $\Phi_{\rho}$. Define a piecewise linear function $\bar{l}$ on the fan $\bar{\Delta}$ as follows

$$
\bar{l}(x)= \begin{cases}l(x) & \text { if } x \in|\Delta| \\ l_{\rho}(p(x)) & \text { if } x \in\left|\Delta^{\prime}\right| .\end{cases}
$$

Then $\bar{l}$ is strictly convex on $\bar{\Delta}$. Indeed, this follows from the inequality $l(x)>\hat{l}(x)=l_{\rho}(p(x))$ for $x$ in the interior of $|\Delta|$.

Proposition 9.4. The map

$$
\bar{l}^{k}: I H^{n-k}(\bar{\Delta}) \rightarrow I H^{n+k}(\bar{\Delta})
$$

is an isomorphism for all $k \geq 1$. That is the (HL) theorem holds for the fan $\bar{\Delta}$ with the strictly convex function $\bar{l}$.

Proof. Let $h \in I H^{n-k}(\bar{\Delta})$ be in the kernel of $\bar{l}^{k}$.

Consider the exact sequence of free $A$-modules

$$
0 \rightarrow \Gamma_{\Delta^{0}} \mathcal{L}_{\bar{\Delta}} \rightarrow \Gamma\left(\mathcal{L}_{\bar{\Delta}}\right) \rightarrow \Gamma\left(\Delta^{\prime}, \mathcal{L}_{\bar{\Delta}}\right) \rightarrow 0
$$

and the induced exact sequence of graded vector spaces

$$
0 \rightarrow I H(\Delta, \partial \Delta) \rightarrow I H(\bar{\Delta}) \rightarrow I H\left(\Delta^{\prime}\right) \rightarrow 0 .
$$

Note that $\bar{\Delta}^{s} \subset[\sigma] \subset \Delta$. Hence by Corollary 8.2, $h \in I H(\Delta, \partial \Delta)$. Since multiplication by $\bar{l}$ is a self adjoint operator with respect to the pairing $(\cdot, \cdot)_{\bar{\Delta}}$ it suffices to prove the following lemma.

Lemma 9.5. The map

$$
l^{k}: I H^{n-k}(\Delta) \rightarrow I H^{n+k}(\Delta)
$$

is surjective for all $k \geq 1$.

Proof of lemma. Let $d=\operatorname{dim}(\sigma)$ and consider the projection $r: V \rightarrow$ $V /\langle\sigma\rangle$. Then $\Phi_{\sigma}=r(\operatorname{Link}(\sigma))$ is a complete fan in $V /\langle\sigma\rangle$. Changing $l$ by a global linear function we may assume that $\left.l\right|_{\Delta}=r^{*}\left(l_{\sigma}\right)$ for a strictly convex function $l_{\sigma}$ on $\Phi_{\sigma}$. Then Lemma 3.18 implies that

$$
I H(\Delta) \simeq I H\left(\Phi_{\sigma}\right) \otimes \overline{\mathcal{L}_{\Delta, \sigma}},
$$

where $l$ acts as $l_{\sigma} \otimes i d$. The assertion of the lemma follows immediately from the (HL) theorem for $\Phi_{\sigma}$ and the fact that the graded vector 
space $\overline{\mathcal{L}_{\Delta, \sigma}}$ is zero in degrees $\geq d(3.13)$. This proves the lemma and the proposition.

Lemma 9.6. The quadratic form $Q_{\bar{l}}$ on $I H(\bar{\Delta})$ satisfies the HodgeRiemann bilinear relations.

Proof. Choose a subspace $V_{1} \subset V$ complementary to $\langle\sigma\rangle$ and identify $V_{1}=V /\langle\sigma\rangle$ by the projection $r$, so that $\Phi_{\sigma}$ is a complete fan in $V_{1}$. Denote by $\overline{[\sigma]} \subset \bar{\Delta}$ the subfan lying in the subspace $\langle\sigma\rangle$. Then $\overline{[\sigma]}$ is a complete subfan in $\langle\sigma\rangle$, whose 1-dimensional cones are those of $\sigma$ and $\rho^{\prime}$.

The projection $r: V \rightarrow V_{1}$ defines a pl-isomorphism of fans $\bar{\Delta} \rightarrow$ $\Phi_{\sigma} \times \overline{[\sigma]}$. Let $\phi: \Phi_{\sigma} \times \overline{[\sigma]} \rightarrow \bar{\Delta}$ be the inverse isomorphism. Then $\phi$ is determined by a function $g: V_{1} \rightarrow\langle\sigma\rangle$ which is piecewise linear with respect to the fan $\Phi_{\sigma}(\operatorname{Link}(\sigma)$ is the graph of $g)$. For each $0 \leq t \leq 1$ the function $\mathrm{tg}$ defines similarly a pl-isomorphism

$$
\phi_{t}: \Phi_{\sigma} \times \overline{[\sigma]} \rightarrow \bar{\Delta}_{t}
$$

Where $\bar{\Delta}_{1}=\bar{\Delta}$ and $\bar{\Delta}_{0}=\Phi_{\sigma} \times \overline{[\sigma]}$. Thus $\Phi_{\sigma} \times \overline{[\sigma]}$ and $\bar{\Delta}$ are two members of a continuous family of fans $\bar{\Delta}_{t}$.

As in the proof of last lemma we can assume that $\left.\bar{l}\right|_{\sigma}=0$ and hence $\left.\bar{l}\right|_{\Delta}=r^{*} l_{\sigma}$ for a strictly convex function $l_{\sigma}$ on $\Phi_{\sigma}$. We write

$$
\bar{l}=r^{*} l_{\sigma}+\left(\bar{l}-r^{*} l_{\sigma}\right),
$$

where $\left(\bar{l}-r^{*} l_{\sigma}\right)$ is supported on $\Delta^{\prime}$. Note that the restriction of $\left(\bar{l}-r^{*} l_{\sigma}\right)$ to $\overline{[\sigma]}$ is strictly convex. Consider the piecewise linear function

$$
\phi^{*} \bar{l}=\phi^{*} r^{*} l_{\sigma}+\phi^{*}\left(\bar{l}-r^{*} l_{\sigma}\right)
$$

on $\Phi_{\sigma} \times \overline{[\sigma]}$. The two summands are the pullbacks of strictly convex functions from $\Phi_{\sigma}$ and $\overline{[\sigma]}$ respectively. For each $0 \leq t \leq 1$ consider the piecewise linear function

$$
\bar{l}_{t}:=\phi_{t}^{-1 *} \phi^{*} \bar{l} .
$$

This function is strictly convex on $\bar{\Delta}_{t}$. Repeating the proof of Proposition 9.4 we find that (HL) theorem holds for the operators $\bar{l}_{t}$ on $I H\left(\bar{\Delta}_{t}\right)$. Thus we obtain a continuous family of graded vector spaces $\operatorname{IH}\left(\bar{\Delta}_{t}\right)$ with continuously varying operators $\bar{l}_{t}$. By the (HL)-property the corresponding quadratic forms $Q_{\bar{l}_{t}}$ have full rank. By Theorem 10.8 the form $Q_{\bar{l}_{0}}$ satisfies the Hodge-Riemann relations. Hence so do all the forms $Q_{\bar{l}_{t}}$. This proves the (HR) theorem for $\bar{\Delta}$.

To complete the proof of (HR) theorem for $\Phi$ we will deduce it from the same theorem for $\Psi$ and $\bar{\Delta}$. 
The fans $\Phi$ and $\bar{\Delta}$ have a common subfan $\Delta$. Define the $A$-module

$$
F:=\left\{\left(s_{1}, s_{2}\right) \in \Gamma\left(\mathcal{L}_{\Phi}\right) \times \Gamma\left(\mathcal{L}_{\bar{\Delta}}\right)\left|\quad s_{1}\right|_{\Delta}=\left.s_{2}\right|_{\Delta}\right\}
$$

with the operator $l_{F}=(l, \bar{l})$ and the bilinear form

$$
[\cdot, \cdot]_{F}: F \times F \rightarrow A(2 n), \quad[\cdot, \cdot]_{F}=[\cdot, \cdot]_{\Phi}-[\cdot, \cdot]_{\bar{\Delta}} .
$$

Lemma 9.7. There exists a morphism of $A$-modules $\beta: F \rightarrow \Gamma\left(\mathcal{L}_{\Psi}\right)$, such that

a) $\beta \cdot l_{F}=\hat{l} \cdot \beta$;

b) $[a, b]_{F}=[\beta(a), \beta(b)]_{\Psi}$.

Proof. By Lemma 3.18 applied to the fans $\hat{\Delta}$ and $\Delta^{\prime}$ and the projection $p: V \rightarrow \bar{V}$ we obtain natural isomorphisms of $A$-modules (also of $\Gamma\left(\mathcal{L}_{\Phi_{\rho}}\right)$-modules $)$

$$
\Gamma\left(\mathcal{L}_{\Delta^{\prime}}\right) \simeq A \otimes_{B} \Gamma\left(\mathcal{L}_{\Phi_{\rho}}\right) \simeq \Gamma\left(\mathcal{L}_{\hat{\Delta}}\right)
$$

This defines a natural isomorphism $\gamma: \Gamma\left(\mathcal{L}_{\Delta^{\prime}}\right) \rightarrow \Gamma\left(\mathcal{L}_{\hat{\Delta}}\right)$.

Consider the exact sequence of $A$-modules

$$
0 \rightarrow \Gamma\left(\mathcal{L}_{\Psi}\right) \rightarrow \Gamma\left(\Psi-\hat{\Delta}^{0}, \mathcal{L}_{\Psi}\right) \oplus \Gamma\left(\hat{\Delta}, \mathcal{L}_{\Psi}\right) \stackrel{(+,-)}{\rightarrow} \Gamma\left(\partial \hat{\Delta}, \mathcal{L}_{\Psi}\right) \rightarrow 0
$$

Define $\beta: \Gamma\left(\mathcal{L}_{\Phi}\right) \oplus \Gamma\left(\mathcal{L}_{\bar{\Delta}}\right) \rightarrow \Gamma\left(\Psi-\hat{\Delta}^{0}, \mathcal{L}_{\Psi}\right) \oplus \Gamma\left(\mathcal{L}_{\hat{\Delta}}\right)$ to be

$$
\beta\left(s_{1}, s_{2}\right):=\left(\left.s_{1}\right|_{\Phi-\Delta^{0}}, \gamma\left(\left.s_{2}\right|_{\Delta^{\prime}}\right)\right) .
$$

This map descends to a map

$$
\beta: F \rightarrow \Gamma\left(\mathcal{L}_{\Psi}\right) .
$$

This is a map of $A$-modules which satisfies the property a) by the choice of functions $\bar{l}$ and $\hat{l}$. Let us show that it satisfies b).

Consider the composition of natural maps

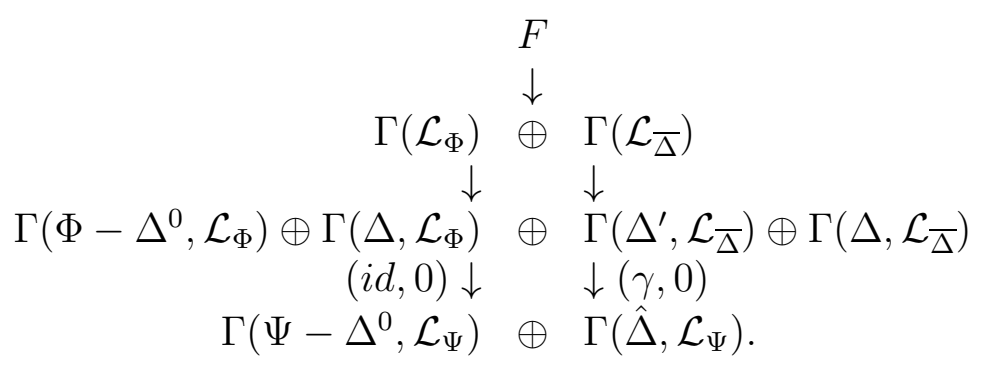

The image of $F$ under this composition is contained in $\Gamma\left(\mathcal{L}_{\Psi}\right)$ (and the composition itself is equal to $\beta$ ). It is convenient to use the pairing $\{\cdot, \cdot\}$ (8.3) on each summand in this diagram. More precisely, on the summands involving $\mathcal{L}_{\bar{\Delta}}$ we take the pairing $\{\cdot, \cdot\}$ with the negative sign. It then suffices to prove that the composition of maps is an isometry. The first map is such by definition. The second is by Proposition 
8.3. The third map is not an isometry, but it is such on the image of $F$. This proves the lemma.

Let $\bar{F}$ as usual denote the graded vector space $F / A^{+} F$. Denote by

$$
(\cdot, \cdot)_{F}: \bar{F} \times \bar{F} \rightarrow \mathbb{R}(2 n)
$$

the residue of the pairing $[\cdot, \cdot]_{F}$. There is a natural map $\bar{F} \rightarrow I H(\Phi) \oplus$ $I H(\bar{\Delta})$.

Lemma 9.8. Let $a \in \operatorname{Prim}_{l} I H^{n-k}(\Phi)$. Then there exists $b \in \operatorname{Prim}_{\bar{l}} I H^{n-k}(\bar{\Delta})$ such that $(a, b)$ belongs to the image of $\bar{F}$.

Proof. Denote by $c \in I H^{n-k}(\Delta)$ the image of $a$. Then $l^{k+1} c=0$. We need to find $b \in \operatorname{Prim}_{\bar{l}} I H^{n-k}(\bar{\Delta})$ such that $\left.b\right|_{\Delta}=c$. Consider the exact sequence

$$
0 \rightarrow I H\left(\Delta^{\prime}, \partial \Delta^{\prime}\right) \rightarrow I H(\bar{\Delta}) \rightarrow I H(\Delta) \rightarrow 0 .
$$

Let $b^{\prime} \in I H^{n-k}(\bar{\Delta})$ be any preimage of $c$. Then $\bar{l}^{k+1} b^{\prime} \in I H^{n+k+2}\left(\Delta^{\prime}, \partial \Delta^{\prime}\right)$. Note that the action of the operator $\left.\bar{l}\right|_{\Delta^{\prime}}$ on $I H\left(\Delta^{\prime}\right)$ coincides with the action of $l_{\rho}$ on $I H\left(\Phi_{\rho}\right)$ (3.18). Hence by the induction hypothesis for $\Phi_{\rho}$ the map

$$
l^{k+1}: I H^{n-k-2}\left(\Delta^{\prime}\right) \rightarrow I H^{n+k}\left(\Delta^{\prime}\right)
$$

is an isomorphism. By Corollary 7.9 the $\mathcal{A}\left(\Delta^{\prime}\right)$-modules $I H\left(\Delta^{\prime}\right)$ and $I H\left(\Delta^{\prime}, \partial \Delta^{\prime}\right)$ are isomorphic with a shift by 2 . Hence the map

$$
\bar{l}^{k+1}: I H^{n-k}\left(\Delta^{\prime}, \partial \Delta^{\prime}\right) \rightarrow I H^{n+k+2}\left(\Delta^{\prime}, \partial \Delta^{\prime}\right)
$$

is an isomorphism. So there exists $h \in I H^{n-k}\left(\Delta^{\prime}, \partial \Delta^{\prime}\right)$ such that $\bar{l}^{k+1} h=\bar{l}^{k+1} b^{\prime}$. Now put $b=b^{\prime}-h$.

Now the (HR) theorem for the fan $\Phi$ follows. Indeed, take $a \in$ $\operatorname{Prim}_{l} I H^{n-k}(\Phi)$. Let $b \in \operatorname{Prim}_{\bar{l}} I H^{n-k}(\bar{\Delta})$ be as in last lemma. Then by Lemma 9.7

$$
\begin{gathered}
(-1)^{\frac{n-k}{2}}\left(a, l^{k} a\right)_{\Phi}-(-1)^{\frac{n-k}{2}}\left(b, \bar{l}^{k} b\right)_{\bar{\Delta}} \\
=(-1)^{\frac{n-k}{2}}\left((a, b), l_{F}(a, b)\right)_{F}=(-1)^{\frac{n-k}{2}}(\beta(a, b), \hat{l} \beta(a, b))_{\Psi} .
\end{gathered}
$$

By the (HR) theorem for $\bar{\Delta}$ and $\Psi$ it follows that

$$
(-1)^{\frac{n-k}{2}}\left(a, l^{k} a\right)_{\Phi} \geq 0 .
$$

Assume that $a \neq 0$. If $b \neq 0$ then

$$
(-1)^{\frac{n-k}{2}}\left(a, l^{k} a\right)_{\Phi} \geq(-1)^{\frac{n-k}{2}}\left(b, l^{k} b\right)_{\bar{\Delta}}>0
$$

and we are done. Assume that $b=0$. Then $\left.a\right|_{\Delta}=0$, and hence $a \in I H\left(\Phi-\Delta^{0}\right)$, so that $\beta(a, b) \neq 0$. Thus

$$
(-1)^{\frac{n-k}{2}}\left(a, l^{k} a\right)_{\Phi}=(-1)^{\frac{n-k}{2}}(\beta(a, b), \hat{l} \beta(a, b))_{\Psi}>0 .
$$


This completes the proof.

\section{KÜNNETH FORMUla FOR $I H$ AND DUALITY ON THE PRODUCT OF FANS}

1. Künneth formula. Let $V_{1}, V_{2}$ be vector spaces of dimensions $n_{1}$ and $n_{2}$ respectively. Let $A_{1}=\operatorname{Sym} V_{1}^{*}, A_{2}=\operatorname{Sym} V_{2}^{*}$ be the (evenly graded) algebras of polynomial functions on $V_{1}$ and $V_{2}$ respectively. Put $V=V_{1} \times V_{2}, A=\operatorname{Sym} V^{*}$; then $A=A_{1} \otimes A_{2}$. Denote by $V_{1} \stackrel{p_{1}}{\leftarrow} V \stackrel{p_{2}}{\rightarrow} V_{2}$ the two projections.

Let $\Delta$ and $\Sigma$ be fans in $V_{1}$ and $V_{2}$ respectively. Consider the product fan $\Phi=\Delta \times \Sigma$ in $V$

$$
\Phi=\{\sigma+\tau \mid \sigma \in \Delta, \tau \in \Sigma\}
$$

with the projections $p_{1}: \Phi \rightarrow \Delta, p_{2}: \Phi \rightarrow \Sigma$.

Let $F_{1} \in \operatorname{Sh}(\Delta), F_{2} \in \operatorname{Sh}(\Sigma)$. Then for every $\sigma \in \Delta$

$$
\left(p_{1 *}\left(p_{1}^{-1} F_{1} \otimes p_{2}^{-1} F_{2}\right)\right)_{\sigma}=F_{1, \sigma} \otimes \Gamma\left(\Sigma, F_{2}\right) .
$$

In particular,

$$
\Gamma\left(\Phi, p_{1}^{-1} F_{1} \otimes p_{2}^{-1} F_{2}\right)=\Gamma\left(\Delta, F_{1} \otimes \Gamma\left(\Sigma, F_{2}\right)\right)=\Gamma\left(\Delta, F_{1}\right) \otimes \Gamma\left(\Sigma, F_{2}\right) .
$$

Note the canonical isomorphisms of sheaves

$$
A_{\Phi}=p_{1}^{-1} A_{\Delta} \otimes p_{2}^{-1} A_{\Sigma}, \quad \mathcal{A}_{\Phi}=p_{1}^{-1} \mathcal{A}_{\Delta} \otimes p_{2}^{-1} \mathcal{A}_{\Sigma} .
$$

In particular we have $\mathcal{A}(\Phi)=\mathcal{A}(\Delta) \otimes \mathcal{A}(\Sigma)$.

Consider the $\mathcal{A}_{\Phi}$-module $\mathcal{L}_{\Phi}^{\prime}:=p_{1}^{-1} \mathcal{L}_{\Delta} \otimes p_{2}^{-1} \mathcal{L}_{\Sigma}$.

Lemma 10.1. There exists a canonical isomorphism of $\mathcal{A}_{\Phi}$-modules $\mathcal{L}_{\Phi}^{\prime}=\mathcal{L}_{\Phi}$, hence an isomorphism $\Gamma\left(\Phi, \mathcal{L}_{\Phi}\right)=\Gamma\left(\Delta, \mathcal{L}_{\Delta}\right) \otimes \Gamma\left(\Sigma, \mathcal{L}_{\Sigma}\right)$. Thus if $\Delta$ and $\Sigma$ are quasi-convex, then so is $\Phi$, and $I H(\Phi)=I H(\Delta) \otimes$ $I H(\Sigma)$.

Proof. Clearly, $\mathcal{L}_{\Phi, \underline{o}}^{\prime}=\mathbb{R}$ and the $\mathcal{A}_{\Phi}$-module $\mathcal{L}_{\Phi}^{\prime}$ is locally free. It suffices to show that the map $\overline{\mathcal{L}_{\Phi, \xi}^{\prime}} \rightarrow \overline{\Gamma\left(\partial \xi, \mathcal{L}_{\Phi}^{\prime}\right)}$ is an isomorphism for $\xi \in \Phi$.

Let $\xi=\sigma+\tau \in \Phi$ where $\sigma \in \Delta, \tau \in \Sigma$. Then $\partial \xi=[\sigma] \times \partial \tau \cup \partial \sigma \times[\tau]$ and

$\Gamma\left(\partial \xi, \mathcal{L}_{\Phi}^{\prime}\right)=\operatorname{Ker}\left\{\Gamma\left([\sigma] \times \partial \tau, \mathcal{L}_{\Phi}^{\prime}\right) \oplus \Gamma\left(\partial \sigma \times[\tau], \mathcal{L}_{\Phi}^{\prime}\right) \stackrel{(+,-)}{\rightarrow} \Gamma\left(\partial \sigma \times \partial \tau, \mathcal{L}_{\Phi}^{\prime}\right)\right\}$.

Note that the complex

$$
\Gamma\left([\xi], \mathcal{L}_{\Phi}^{\prime}\right) \rightarrow \Gamma\left([\sigma] \times \partial \tau, \mathcal{L}_{\Phi}^{\prime}\right) \oplus \Gamma\left(\partial \sigma \times[\tau], \mathcal{L}_{\Phi}^{\prime}\right) \stackrel{(+,-)}{\rightarrow} \Gamma\left(\partial \sigma \times \partial \tau, \mathcal{L}_{\Phi}^{\prime}\right)
$$

is isomorphic to the tensor product of complexes

$$
(*) \quad \Gamma\left([\sigma], \mathcal{L}_{\Delta}\right) \rightarrow \Gamma\left(\partial \sigma, \mathcal{L}_{\Delta}\right), \quad \Gamma\left([\tau], \mathcal{L}_{\Sigma}\right) \rightarrow \Gamma\left(\partial \tau, \mathcal{L}_{\Sigma}\right)
$$


It follows that the map

$$
\Gamma\left([\xi], \mathcal{L}_{\Phi}^{\prime}\right) \rightarrow \Gamma\left(\partial \xi, \mathcal{L}_{\Phi}^{\prime}\right)
$$

is surjective (i.e. the sheaf $\mathcal{L}_{\Phi}^{\prime}$ is flabby), hence also the map $\overline{\mathcal{L}_{\Phi, \xi}^{\prime}} \rightarrow$ $\overline{\Gamma\left(\partial \xi, \mathcal{L}_{\Phi}^{\prime}\right)}$ is such. The two complexes $(*)$ (and so their tensor product) become acyclic after taking the residue at the maximal ideal $A^{+} \subset A$. It follows that the map $\overline{\mathcal{L}_{\Phi, \xi}^{\prime}} \rightarrow \overline{\Gamma\left(\partial, \mathcal{L}_{\Phi}^{\prime}\right)}$ is also injective.

Lemma 10.2. Assume that the fans $\Delta$ and $\Sigma$ (hence also $\Phi$ ) are quasiconvex. Then

$$
\Gamma_{\Phi^{0}} \mathcal{L}_{\Phi}=\Gamma_{\Delta^{0}} \mathcal{L}_{\Delta} \otimes \Gamma_{\Sigma^{0}} \mathcal{L}_{\Sigma}
$$

Hence $I H(\Phi, \partial \Phi)=I H(\Delta, \partial \Delta) \otimes I H(\Sigma, \partial \Sigma)$.

Proof. By taking the tensor product of short exact sequences

$$
0 \rightarrow \Gamma_{\Delta^{0}} \mathcal{L}_{\Delta} \rightarrow \Gamma\left(\mathcal{L}_{\Delta}\right) \rightarrow \Gamma\left(\partial \Delta, \mathcal{L}_{\Delta}\right) \rightarrow 0,
$$

and

$$
0 \rightarrow \Gamma_{\Sigma^{0}} \mathcal{L}_{\Sigma} \rightarrow \Gamma\left(\mathcal{L}_{\Sigma}\right) \rightarrow \Gamma\left(\partial \Sigma, \mathcal{L}_{\Sigma}\right) \rightarrow 0
$$

we find the exact sequence

$$
\begin{gathered}
0 \rightarrow \Gamma_{\Delta^{0}} \mathcal{L}_{\Delta} \otimes \Gamma_{\Sigma^{0}} \mathcal{L}_{\Sigma} \rightarrow \Gamma\left(\mathcal{L}_{\Delta}\right) \otimes \Gamma\left(\mathcal{L}_{\Sigma}\right) \\
\rightarrow\left[\Gamma\left(\partial \Delta, \mathcal{L}_{\Delta}\right) \otimes \Gamma\left(\mathcal{L}_{\Sigma}\right)\right] \oplus\left[\Gamma\left(\mathcal{L}_{\Delta}\right) \otimes \Gamma\left(\partial \Sigma, \mathcal{L}_{\Sigma}\right)\right] .
\end{gathered}
$$

Note that the kernel of the map

$$
\Gamma\left(\mathcal{L}_{\Delta}\right) \otimes \Gamma\left(\mathcal{L}_{\Sigma}\right) \rightarrow\left[\Gamma\left(\partial \Delta, \mathcal{L}_{\Delta}\right) \otimes \Gamma\left(\mathcal{L}_{\Sigma}\right)\right] \oplus\left[\Gamma\left(\mathcal{L}_{\Delta}\right) \otimes \Gamma\left(\partial \Sigma, \mathcal{L}_{\Sigma}\right)\right]
$$

is naturally isomorphic to the kernel of the map

$$
\Gamma\left(\mathcal{L}_{\Phi}\right) \rightarrow \Gamma\left(\partial \Phi, \mathcal{L}_{\Phi}\right)
$$

Hence

$$
\Gamma_{\Phi^{0}} \mathcal{L}_{\Phi}=\Gamma_{\Delta^{0}} \mathcal{L}_{\Delta} \otimes \Gamma_{\Sigma^{0}} \mathcal{L}_{\Sigma}
$$

2. Duality on the product of fans. Consider the dualizing modules $\omega_{1}=A_{1} \cdot \wedge^{n_{1}} V_{1}^{*}, \omega_{2}=A_{2} \cdot \wedge^{n_{2}} V_{2}^{*}$ on $V_{1}$ and $V_{2}$. Then $\omega=\omega_{1} \otimes \omega_{2}$.

Choose volume forms $\Omega_{V_{i}} \in \wedge^{n_{i}} V_{i}^{*}, i=1,2$ and put $\Omega_{V}=\Omega_{V_{1}} \wedge \Omega_{V_{2}}$.

Assume that orientations of cones in $\Delta$ and $\Sigma$ are chosen (the cones of top dimension are oriented by $\Omega_{V_{1}}$ and $\Omega_{V_{2}}$ respectively). This determines the cellular complexes $C_{\Delta}^{\bullet}(\cdot)$ and $C_{\Sigma}^{\bullet}(\cdot)$. Given $\xi=\sigma+\tau \in \Phi$ with $\sigma \in \Delta, \tau \in \Sigma$ we orient it in the usual way by putting first the vectors in $\sigma$ and then the vectors in $\tau$. Thus the cones of dimension $n$ in $\Phi$ are oriented by $\Omega_{V}$. This defines the cellular complex $C_{\Phi}^{\bullet}(\cdot)$. Note 
that $C_{\Phi}^{\bullet}=C_{\Delta}^{\bullet} \otimes C_{\Sigma}^{\bullet}$ as graded vector spaces, but not as complexes. I.e. for $F_{1} \in \operatorname{Sh}(\Delta), F_{2} \in \operatorname{Sh}(\Sigma), F=p_{1}^{-1} F_{1} \otimes p_{2}^{-1} F_{2} \in \operatorname{Sh}(\Phi)$ we have

$$
C_{\Phi}^{k}(F)=\bigoplus_{s} C_{\Delta}^{s}\left(F_{1}\right) \otimes C_{\Sigma}^{k-s}\left(F_{2}\right)
$$

and the differential on the right is

$$
d(a \otimes b)=d(a) \otimes b+(-1)^{s} a \otimes d(b), \quad \text { for } a \otimes b \in C_{\Delta}^{s}\left(F_{1}\right) \otimes C_{\Sigma}^{t}\left(F_{2}\right),
$$

whereas on the left it is

$$
d(a \otimes b)=d(a) \otimes b+(-1)^{n_{1}+s} a \otimes d(b) .
$$

To fix this discrepancy let us consider the new cellular complex $\bar{C}^{\bullet}$ which is equal to the shifted (to the left) complex $C^{\bullet}[d]$, except it has the same differential (and not $(-1)^{d}$ times the original one), where $d$ is the dimension of the ambient space. Then the identification

$$
\bar{C}_{\Phi}^{\bullet}=\bar{C}_{\Delta}^{\bullet} \otimes \bar{C}_{\Sigma}^{\bullet}
$$

is the equality of complexes.

Note that we can use the complex $\bar{C}^{\bullet}$ instead of $C^{\bullet}$ in Proposition 3.9. Namely for $F \in D_{c}^{b}\left(A_{\Phi}-\bmod \right)$ the functor $\bar{C}_{\Phi}^{\bullet}$ induces the isomorphism

$$
\mathbb{R} \Gamma(\Phi, D F) \simeq \mathbb{R} \operatorname{Hom}_{A}^{\bullet}\left(\bar{C}^{\bullet}, \omega[n]\right),
$$

and similarly for $\Delta$ and $\Sigma$. We will use this description of the duality to show that it is compatible with the product of fans.

Let $P_{1}^{\bullet} \in D_{c}^{b}\left(A_{1}-\bmod \right), P_{2}^{\bullet} \in D_{c}^{b}\left(A_{2}-\bmod \right), P^{\bullet}=P_{1}^{\bullet} \otimes P_{2}^{\bullet} \in$ $D_{c}^{b}(A-\bmod )$. Then there exists a natural functorial isomorphism in $D_{c}^{b}(A-\bmod )$ :

$\delta: \mathbb{R} \operatorname{Hom}_{A_{1}}\left(P_{1}^{\bullet}, \omega_{1}\left[n_{1}\right]\right) \otimes \mathbb{R} \operatorname{Hom}_{A_{2}}\left(P_{2}^{\bullet}, \omega_{2}\left[n_{2}\right]\right) \rightarrow \mathbb{R} \operatorname{Hom}_{A}\left(P^{\bullet}, \omega[n]\right)$,

$$
\delta(f \otimes g)(a \otimes b)=(-1)^{\operatorname{deg}(g) \operatorname{deg}(a)} f(a) \otimes g(b) .
$$

Example 10.3. In the previous notation let $P_{1}^{\bullet}=\mathbb{R}=P_{2}^{\bullet}$. As was remarked in the proof of Lemma 3.15 above we have canonical isomorphisms $\operatorname{Ext}_{A}^{n}(\mathbb{R}, \omega[n])=\mathbb{R}=\operatorname{Ext}_{A_{i}}^{n_{i}}\left(\mathbb{R}, \omega_{i}\left[n_{i}\right]\right)$ for $i=1,2$. The map $\delta$ induces an isomorphism

$$
\delta: \operatorname{Ext}_{A_{1}}^{n_{1}}\left(\mathbb{R}, \omega_{1}\left[n_{1}\right]\right) \otimes \operatorname{Ext}_{A_{2}}^{n_{2}}\left(\mathbb{R}, \omega_{2}\left[n_{2}\right]\right) \rightarrow \operatorname{Ext}_{A}^{n}(\mathbb{R}, \omega[n])
$$

which coincides with the multiplication map $\mathbb{R} \otimes \mathbb{R} \rightarrow \mathbb{R}$ under the above isomorphisms. (Indeed, the tensor product of the Koszul resolutions of $\mathbb{R}$ as $A_{1^{-}}$and $A_{2}$-module respectively is equal to its Koszul resolution as an $A$-module.) 
Proposition 10.4. a) Let $F_{1} \in D_{c}^{b}\left(A_{\Delta}-\bmod \right), F_{2} \in D_{c}^{b}\left(A_{\Sigma}-\bmod \right)$ and $F=p_{1}^{-1} F_{1} \otimes p_{2}^{-1} F_{2} \in D_{C}^{b}\left(A_{\Phi}-\right.$ mod $)$. The map $\delta$ defines a functorial isomorphism in $D_{c}^{b}\left(A_{\Phi}-\bmod \right)$

$$
\delta: p_{1}^{-1} D F_{1} \otimes p_{2}^{-1} D F_{2} \rightarrow D F .
$$

b) In case $F_{1}=\mathcal{L}_{\Delta}, F_{2}=\mathcal{L}_{\Sigma}$ and $F=\mathcal{L}_{\Phi}$ this isomorphism has the following properties:

i) If we use the canonical identifications of stalks $D(\mathcal{L})_{\underline{o}}=\mathbb{R}$ on each of the three fans, then $\delta_{\underline{o}}$ is the multiplication map $\mathbb{R} \otimes \mathbb{R} \rightarrow \mathbb{R}$.

ii) Assume that the fans $\Delta, \Sigma$ (and hence $\Phi$ ) are quasi-convex. We use isomorphisms of Proposition 3.9 (with $\bar{C}^{\bullet}$ instead of $C^{\bullet}$ ) and Lemma 10.2. Then on the level of global sections the map

$$
\delta: \operatorname{Hom}_{A_{1}}\left(\Gamma_{\Delta^{0}} \mathcal{L}_{\Delta}, \omega_{1}\right) \otimes \operatorname{Hom}_{A_{2}}\left(\Gamma_{\Sigma^{0}} \mathcal{L}_{\Sigma}, \omega_{2}\right) \rightarrow \operatorname{Hom}_{A}\left(\Gamma_{\Phi^{0}} \mathcal{L}_{\Phi}, \omega\right)
$$

is given by

$$
\delta(f \otimes g)(a \otimes b)=f(a) \otimes g(b) .
$$

Proof. a) Let $\xi=\sigma+\tau \in \Phi$. It follows from Proposition 3.9 that $D F([\xi])=\mathbb{R} \operatorname{Hom}_{A}\left(\bar{C}_{\Phi}^{*}\left(F_{[\xi]}\right), \omega[n]\right)$ and similarly for $D F_{1}([\sigma])$ and $D F_{2}([\tau])$. Applying $\delta$ to the complexes $\bar{C}_{\Phi}^{\bullet}\left(F_{[\xi]}\right)=\bar{C}_{\Delta}^{\bullet}\left(F_{1[\sigma]}\right) \otimes \bar{C}_{\Sigma}^{\bullet}\left(F_{2[\tau]}\right)$ we obtain the required functorial isomorphism

$$
\delta: p_{1}^{-1} D F_{1} \otimes p_{2}^{-1} D F_{2} \rightarrow D F .
$$

b) i) follows from Example 10.3, and ii) follows from the explicit formula for the morphism $\delta$.

Consider the diagram of sheaves on $\Phi$

$$
\begin{array}{ccccc}
p_{1}^{-1} \mathcal{L}_{\Delta} & \otimes & p_{2}^{-1} \mathcal{L}_{\Sigma} & = & \mathcal{L}_{\Phi} \\
p_{1}^{-1} \epsilon_{\Delta} \downarrow & & \downarrow p_{2}^{-1} \epsilon_{\Sigma} & & \downarrow \epsilon_{\Phi} \\
p_{1}^{-1} D \mathcal{L}_{\Delta} \otimes & p_{2}^{-1} D \mathcal{L}_{\Sigma} & \stackrel{\delta}{\rightarrow} & D \mathcal{L}_{\Phi} .
\end{array}
$$

Note that all arrows are isomorphisms.

Lemma 10.5. The above diagram commutes.

Proof. The stalks of all the sheaves at the origin $\underline{o}$ are canonically isomorphic to $\mathbb{R}$. It follows from Proposition 10.4 b)i) and the definition of the morphism $\epsilon$ that the diagram commutes at the origin. Hence it commutes by the rigidity of $\mathcal{L}_{\Phi}$.

Proposition 10.6. Let $\Delta$ and $\Sigma$ be quasi-convex fans, $\Phi=\Delta \times \Sigma$. Then the pairing

$$
\Gamma\left(\mathcal{L}_{\Phi}\right) \times \Gamma_{\Phi^{0}} \mathcal{L}_{\Phi} \rightarrow A(2 n)
$$

is equal to the tensor product of the pairings

$$
\Gamma\left(\mathcal{L}_{\Delta}\right) \times \Gamma_{\Delta^{0}} \mathcal{L}_{\Delta} \rightarrow A_{1}\left(2 n_{1}\right),
$$


and

$$
\Gamma\left(\mathcal{L}_{\Sigma}\right) \times \Gamma_{\Sigma^{0}} \mathcal{L}_{\Sigma} \rightarrow A_{2}\left(2 n_{2}\right)
$$

under the isomorphisms

$$
\begin{gathered}
\Gamma\left(\mathcal{L}_{\Phi}\right)=\Gamma\left(\mathcal{L}_{\Delta}\right) \otimes \Gamma\left(\mathcal{L}_{\Sigma}\right) \\
\Gamma_{\Phi^{0}} \mathcal{L}_{\Phi}=\Gamma_{\Delta^{0}} \mathcal{L}_{\Delta} \otimes \Gamma_{\Sigma^{0}} \mathcal{L}_{\Sigma}
\end{gathered}
$$

and

$$
A(2 n)=A_{1}\left(2 n_{1}\right) \otimes A_{2}\left(2 n_{2}\right)
$$

Proof. Applying the functor of global sections to the commutative diagram of Lemma 10.5 we obtain the commutative diagram

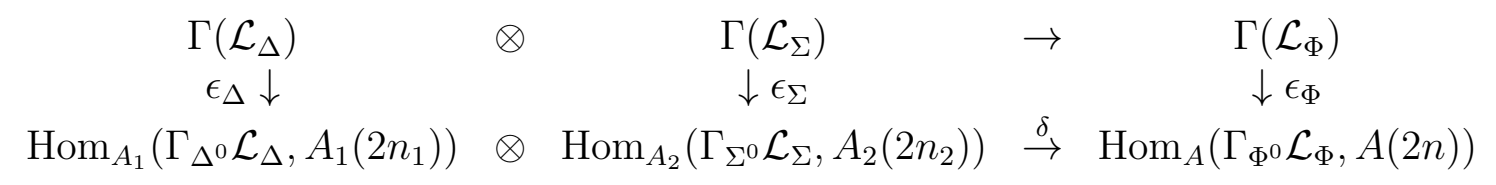

Now apply Proposition 10.4 b) ii).

Corollary 10.7. Let $\Delta$ and $\Sigma$ be quasi-convex fans, $\Phi=\Delta \times \Sigma$. Then the pairing

$$
I H(\Phi) \times I H(\Phi, \partial \Phi) \rightarrow \mathbb{R}(2 n)
$$

is the tensor product of pairings

$$
I H(\Delta) \times I H(\Delta, \partial \Delta) \rightarrow \mathbb{R}\left(2 n_{1}\right)
$$

and

$$
I H(\Sigma) \times I H(\Sigma, \partial \Sigma) \rightarrow \mathbb{R}\left(2 n_{2}\right) .
$$

In particular this holds if the fans are complete.

3. The (HL) and (HR) theorems for product of fans. Let $l_{1}$ and $l_{2}$ be strictly convex piecewise linear functions on the fans $\Delta$ and $\Sigma$ respectively. Then $l=l_{1}+l_{2}$ is strictly convex on $\Phi=\Delta \times \Sigma$.

Theorem 10.8. Assume that fans $\Delta, \Sigma$ (and hence also $\Phi$ ) are complete. If the (HL) (resp. (HR)) theorem holds for operators $l_{1}$ and $l_{2}$ on $I H(\Delta)$ and $I H(\Sigma)$ then it also holds for the operator $l$ on $I H(\Phi)$.

Proof. We have $I H(\Phi)=I H(\Delta) \otimes I H(\Sigma)$ and $l=l_{1} \otimes 1+1 \otimes l_{2}$. The statement about the (HL) theorem follows.

Choose a basis for the primitive parts $\operatorname{Prim}_{l_{1}} I H(\Delta)$ and $\operatorname{Prim}_{l_{2}} I H(\Sigma)$ which is orthogonal with respect to the form $Q_{l_{1}}$ and $Q_{l_{2}}$ respectively. This defines an orthogonal decomposition of $I H(\Delta)$ and $I H(\Sigma)$ into cyclic $\mathbb{R}\left[l_{1}\right]$ and $\mathbb{R}\left[l_{2}\right]$-modules respectively. But for cyclic modules the assertion can be deduces from the classical Hodge-Riemann relations of the primitive cohomology of the product of projective spaces. (It would be nice to have an elementary algebraic proof but we do not have one.) 


\section{REFERENCES}

[Bri] M. Brion, The structure of the polytope algebra, Tôhoku Math. J. 49 (1997), $1-32$.

[BrLu] P. Bressler, V. A. Lunts, Intersection cohomology on nonrational polytopes, e-print math.AG/0002006, to appear in Compositio Math.

[BeLu] J. Bernstein, V. Lunts, Equivariant Sheaves and Functors, LNM 1578, Springer-Verlag, 1994.

[McM] P. McMullen, On simple polytopes, Invent. math. 113, 419-444 (1993).

[S] R. Stanley, Generalized h-vectors, intersection cohomology of toric varieties and related results, in Commutative Algebra and Combinatorics, M. Nagata and H. Matsumura, eds., Adv. Stud. Pure Math. 11, Kinokunia, Tokyo, and North-Holland, Amsterdam/New York, 1987, 187-213.

[Ti] V. Timorin, An analogue of Hodge-Riemann bilinear relations for simple convex polytopes, Russian Math. Surveys 54, No. 2, 381-426 (1999).

[BBFK] G. Barthel, J.-P. Brasselet, K.-H. Fiesler and L. Kaup, Combinatorial intersection cohomology for fans, Tohoku Math. J. 54, 1-41 (2002).

[Ka] K. Karu, Hard Lefschetz theorem for nonrational polytopes, e-print math.AG/0112087.

Department of Mathematics, University of Arizona, Tucson, AZ, 85721, USA

E-mail address: bressler@math.arizona.edu

Department of Mathematics, Indiana University, Bloomington, IN 47405, USA

E-mail address: vlunts@indiana.edu 